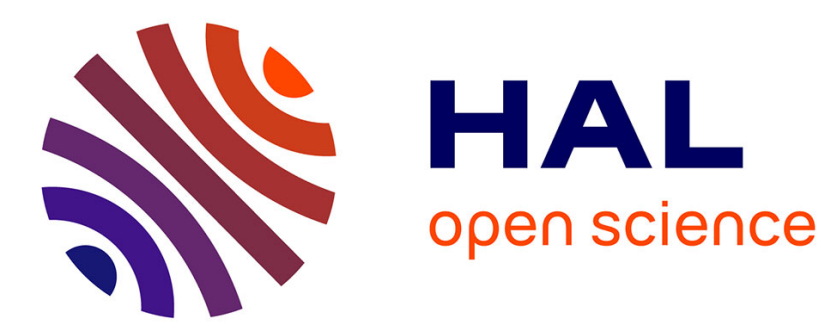

\title{
Silent inflow condition for turbulent boundary layers
}

Xavier Gloerfelt, Jean-Christophe Robinet

\section{To cite this version:}

Xavier Gloerfelt, Jean-Christophe Robinet. Silent inflow condition for turbulent boundary layers. Physical Review Fluids, 2017, 2 (12), pp.124603-124634. 10.1103/PhysRevFluids.2.124603 . hal02445326

\section{HAL Id: hal-02445326 \\ https://hal.science/hal-02445326}

Submitted on 20 Jan 2020

HAL is a multi-disciplinary open access archive for the deposit and dissemination of scientific research documents, whether they are published or not. The documents may come from teaching and research institutions in France or abroad, or from public or private research centers.
L'archive ouverte pluridisciplinaire HAL, est destinée au dépôt et à la diffusion de documents scientifiques de niveau recherche, publiés ou non, émanant des établissements d'enseignement et de recherche français ou étrangers, des laboratoires publics ou privés. 


\title{
Silent inflow condition for turbulent boundary layers
}

\author{
X. Gloerfelt and J.-C. Robinet \\ DynFluid Laboratory, Arts et Métiers ParisTech, 151 Boulevard de l'Hopital, 75013 Paris, France
}

\begin{abstract}
The generation of a turbulent inflow is a tricky problem. In the framework of aeroacoustics, another important constraint is that the numerical strategy used to reach a turbulent state induces a spurious noise which is lower than the acoustic field of interest. For the study of noise radiated directly by a turbulent boundary layer on a flat plate, this constraint is severe since wall turbulence is a very inefficient source. That is why a method based on a transition by modal interaction using a base flow with an inflection point is proposed to cope with that. The base flow must be a solution of the equations so we use a profile behind a backward-facing step representative of experimental trip bands. A triad of resonant waves is selected by a local stability analysis of the linearized compressible equations and is added with a weak amplitude in the inlet plane. The compressible stability calculation allows the specification of the thermodynamic quantities at the inlet, which turns out to be fundamental to ensure a quiet inflow. A smooth transition is achieved with the rapid formation of $\Lambda$-shape vortices in a staggered organization as in subharmonic transition. The dominance of oblique waves promotes a rapid breakdown by the liftup mechanism of low-speed streaks. The quality of the fully turbulent state is assessed and the direct noise radiation from a turbulent boundary layer at Mach 0.5 is obtained with a very low level of spurious noise.
\end{abstract}

\section{INTRODUCTION}

The prescription of silent turbulent inflow boundary conditions for the simulations of spatially inhomogeneous flows is particularly tricky. Several methods for the generation of inflow turbulence have been proposed in the direct numerical and large eddy simulation literature. The challenge is even greater when one considers noise generation by the flow. Direct noise computations (DNC), which consist of obtaining the aerodynamic and acoustic fields in the same simulation, are made possible by the use of appropriate mathematical models and numerical schemes, but also rely heavily on properly chosen boundary conditions. The development of nonreflecting conditions allowing the exit of acoustic waves has been an active field of research in the past years. For DNC, it is furthermore of great importance to make the turbulence coexist with these nonreflecting conditions. The perturbations of the radiation condition due to the flow exit are generally reduced by the addition of a buffer zone. The design of a silent turbulent inflow boundary condition is more cumbersome. A critical discussion on the inflow methods is proposed in Sec. III. Some explorations conducted in the past [1-3] have shown that the issue is interrelated with the numerical choices for the inflow boundary condition and the discretization schemes. Furthermore, for a compressible simulation, it is important to specify correctly the thermodynamic quantities to ensure a quiet inflow.

Some attempts have been made toward the prediction of the noise radiated by a turbulent boundary layer [4]. This is a fundamental source of aerodynamic noise that has also a great practical interest. Turbulent boundary layers can provide an indirect contribution to the noise, by exciting vibrations of the structure, and a direct noise contribution. The latter part can play a significant role even if its intensity is very low, explaining why it is hardly measured unambiguously. Indeed, by virtue of Powell's reflection principle [5], dipolar source terms, associated with the presence of solid boundaries in the acoustic analogy [6], will degenerate toward quadripolar sources when placed near an infinite plane. The implication is that the aeroacoustic sources are rather inefficient, as in free turbulence. This explains why no experiment has been capable hitherto of measuring acoustic 
radiation from a plane boundary layer. DNC has been shown to be a powerful tool to design an ideal numerical experiment [4]. The computation remains expensive, however, and it is important to find an efficient and silent way to obtain a fully developed turbulent boundary layer in this particularly demanding configuration. Boundary layer noise was obtained in Refs. [4,7,8] by using a small step to trigger the transition to turbulence. The small step induced some spurious noise, since a rough wall is known to generate higher noise levels than a smooth wall [9]. A step indeed behaves as a scattering object, leading to a dipolar acoustic field that can dominate the quadrupolar sources associated to the boundary layer, notably as the velocity is decreased. The noise induced by the small step was not too penalizing at Mach 0.5 but it was seen to overwhelm the direct radiation for lower Mach numbers (0.1 to 0.3). Even if the trick used to ignite transition toward a turbulent state has been seen to produce considerably less spurious noise than other inflow conditions based on the introduction of synthetic turbulence or on recycling methods [1,3], further investigations toward an efficient and silent inflow method is needed to minimize inflow acoustic disturbance.

Preliminary studies, where instability modes are superimposed on an inlet inflectional velocity profile, were carried out for a cylindrical cavity flow [3] and for the interaction of an oblique shock with a supersonic turbulent boundary layer [10]. Besides, in previous studies about trailing edge noise [11] or boundary layer noise [4], the transition was triggered by using a two-dimensional step, similar to the tripping devices in experiments. The trick was efficient even if the small step inside the computational domain can behave as a scattering object, resulting in a supplementary dipole source which can be problematic at low speeds. In the present study, both ideas are combined: An inflectional velocity is directly imposed at the inlet and is determined in a previous steady computation behind a backward-facing step. The amplification factor of Kelvin-Helmholtz-like instabilities allows the introduction of very weak disturbances, reducing the possible spurious noise. Moreover, the instability waves are computed from the linearized compressible NavierStokes equations, so that the pressure and density perturbations are known at the inlet, which is a necessary condition to reduce spurious noise. Finally, another ingredient which is beneficial for rapid transition is the modal interaction. That is why a triad of unstable modes, comprising a pair of oblique modes and a two-dimensional mode, is selected from the compressible linear-stability analysis.

The basic idea of our turbulent inflow condition is to reproduce the physical ingredients which explain the rapid turbulent eruption just behind the step without using the step. The paper is organized as follows. In the first section, the numerical algorithm is presented and the importance of initializing correctly the thermodynamic variables in the numerical inflow condition is discussed. The second section gives a brief overview of the numerous methods used for turbulent inflow conditions in order to stress the common pitfalls and the main ingredients we want to include. The developed method is presented in Sec. IV, describing the base flow behind a backward-facing step and the local stability analysis to find an unstable resonant triad. The results by triggering turbulence breakdown with these particular instability waves are analyzed in Sec. V. The physical nature of the transition and the quality of the turbulent state are investigated. Direct radiation from a turbulent boundary layer at Mach number 0.5 is obtained without pollution from spurious noise.

\section{NUMERICAL METHODS FOR DIRECT NOISE CALCULATION}

High-fidelity methods such as direct numerical simulation (DNS) and large eddy simulation (LES) are powerful tools to improve understanding of the noise generation process in specific situations (see reviews in Refs. [12-14]). The numerical strategy should be designed to minimize spurious noise sources that can mask the acoustic radiation under investigation. The global choice of the numerical algorithms has a direct impact on the quality of the inflow condition. Particular attention is devoted to the problem of nonreflecting boundary conditions, which is crucial for the present purpose. 


\section{A. Flow solver}

The full three-dimensional compressible Navier-Stokes equations are solved in a conservative form (see Ref. [4]). The convective terms are integrated in time using an explicit low-storage six-step Runge-Kutta scheme optimized in the wave-number space [15]. The viscous terms are integrated at the last substep, i.e., at first order, since the viscous stability criterion would give a significantly lower time step for viscothermal terms. Spatial derivatives are discretized with optimized finite differences on an 11-point stencil [15] for the convective fluxes, and standard fourth-order finite differences for the viscous and heat fluxes. A one-dimensional coordinate transform is implemented to account for grid stretching, notably used in the wall-normal direction. Geometric stretching rates lower than $2.5 \%$ are used to keep good properties of the optimized finite-difference scheme (see Sec. 7.2 in Ref. [15]). At the last substep of the explicit integration scheme, a selective filtering on an 11-point stencil [15] is incorporated in each direction to eliminate grid-to-grid unresolved oscillations. It is applied with an amplitude of 0.2 [4].

On the wall boundary, the no-slip conditions $u=v=w=0$ are imposed, with $\partial p / \partial n=0$, where $n$ is the direction normal to the solid surface. The finite-difference stencil for the convective terms is progressively reduced down to the second order. The viscous stress terms are evaluated from the interior points by using fourth-order backward differences. The wall temperature is calculated with the adiabatic condition, and the density is deduced from the ideal gas law.

Since a large computational domain is required for wave-number-frequency analyses of wall pressure, the simulations are wall-resolved LES, rather than DNS (see Sec. IV B). The selective filter used to damp unresolved high-frequency waves provides the dissipation necessary to approximately mimic unresolved scales. Appendix B shows that the chosen resolution is sufficient to reproduce an oblique transition scenario with the present code.

\section{B. Radiation boundary conditions}

Numerically, it is often tricky to impose a nonreflecting inflow condition, even if an analytical or numerical solution for the incoming mean flow is already known. The most popular numerical boundary conditions used for that purpose are based on the characteristic formulation [16,17]. The inflow variables are decomposed in the characteristic basis, so that it is possible to specify each characteristic independently. In our aeroacoustic solver, the radiation boundary conditions of Tam and Dong [18,19], using a far-field solution of the sound waves, have been retained since they are very efficient notably in the corners (because they are expressed in cylindrical coordinates, whereas the characteristic are inherently 1D). In all cases, the governing equations are changed in the few first planes parallel to the boundary, and the numerical implementation must be carefully chosen to ensure the best compatibility with interior scheme.

For spatially developing boundary layers, periodic boundary conditions are applied at the lateral boundaries, so that we choose to use a three-dimensional (3D) cylindrical formulation of Tam and Dong's condition:

$$
\left(\frac{1}{V_{g}} \frac{\partial}{\partial t}+\frac{\partial}{\partial r}+\frac{1}{r}\right)\left[\begin{array}{l}
\rho_{a} \\
\mathbf{u}_{a} \\
p_{a}
\end{array}\right]=0,
$$

where $V_{g}$ denotes the group velocity of acoustic waves and is defined by

$$
V_{g}=\overline{\mathbf{u}} \cdot \mathbf{e}_{\mathbf{r}}+\sqrt{\bar{c}^{2}-\left(\overline{\mathbf{u}} \cdot \mathbf{e}_{\theta}\right)^{2}} \text { with }\left\{\begin{array}{l}
\mathbf{e}_{\mathbf{r}}=(\cos \theta, \sin \theta) \\
\mathbf{e}_{\theta}=(-\sin \theta, \cos \theta)
\end{array} .\right.
$$

The subscript $a$ in (1) indicates that only acoustic quantities are considered in the free-field boundaries. These quantities are approximated by $(\rho-\bar{\rho}, \mathbf{u}-\overline{\mathbf{u}}, p-\bar{p})$, where the overbar indicates a temporal mean computed all along the simulation. When vortical structures interact with one of the boundaries, these assumptions are no more verified, and the interaction with the changing mean flow can introduce numerical artifacts. In particular, it is sometimes difficult to maintain the boundary 
condition clean during the transient phase of a simulation. For wall-bounded flows, the radiation condition must also be compatible with the wall condition in the lower corners. Moreover, the radiation conditions (1) are expressed in the polar coordinates $(r, \theta)$, which requires the arbitrary choice of a radiation center (origin of polar coordinates). It has been shown in previous studies [19] that the exact location of the radiation center is not very important if the boundaries are sufficiently far from the sources. For the present case of boundary-layer flows, a pragmatic choice to connect inflow and outflow conditions to the wall is to locate the radiation center on the wall in the median plane. This avoids having group velocity directed toward the wall and reduces artifacts at the lower corners. Furthermore, our aim is to prescribe the mean flow in the inlet boundary without imposing directly the values of $\overline{\mathbf{u}}$, which would result in a partially reflecting condition. For that purpose, the initial condition for $(\bar{\rho}, \overline{\mathbf{u}}, \bar{p})$ must be compatible with the resolved equations in the interior domain, and it is more beneficial to locate the radiation center close to the inlet boundary. To superimpose disturbances in the inlet plane, required to trigger transition toward a fully turbulent state, a triple decomposition is used as proposed by Tam [19]. The modified system for the inflow boundary reads:

$$
\left(\frac{1}{V_{g}} \frac{\partial}{\partial t}+\frac{\partial}{\partial r}+\frac{1}{r}\right) \quad\left[\begin{array}{l}
\rho-\bar{\rho} \\
\mathbf{u}-\overline{\mathbf{u}} \\
p-\bar{p}
\end{array}\right]=\left(\frac{1}{V_{g}} \frac{\partial}{\partial t}+\frac{\partial}{\partial r}+\frac{1}{r}\right)\left[\begin{array}{l}
\rho_{\text {in }} \\
\mathbf{u}_{\text {in }} \\
p_{\text {in }}
\end{array}\right],
$$

where $\mathbf{q}_{\text {in }}^{\prime}=\left(\rho_{\text {in }}^{\prime}, \mathbf{u}_{\text {in }}^{\prime}, p_{\text {in }}^{\prime}\right)^{T}$ are the density, velocity vector, and pressure disturbances to be imposed at the inlet. The right-hand side of (2) is prescribed and is thus a source term in the radiation conditions. One important point to avoid spurious noise when entering the perturbations is to prescribe both a solenoidal velocity field $\mathbf{u}_{\text {in }}$, and the corresponding thermodynamic variables $\rho_{\text {in }}$ and $p_{\text {in }}$.

To illustrate the importance of initializing correctly the thermodynamic variables $p$ and $\rho$ in the inflow planes, the previous formulation (2) is tested for the introduction of a Taylor's vortex, defined as

$$
\left\{\begin{array}{l}
u^{\prime}=A\left(y-y_{0}\right) \exp \left(\Lambda R^{2}\right) \\
v^{\prime}=-A\left(x-x_{0}\right) \exp \left(\Lambda R^{2}\right)
\end{array}, \quad \text { where } \quad R=\sqrt{\left(x-x_{0}\right)^{2}-\left(y-y_{0}\right)^{2}} \quad \text { and } \quad \Lambda=-\ln 2 / b^{2} .\right.
$$

The Gaussian half-width $b$ is taken as $3 \Delta y$. The velocity field is solenoidal and the initial pressure is known analytically from the Poisson problem:

$$
p^{\prime}=-\rho_{\infty} \frac{A^{2}}{4 \Lambda} \exp \left(2 \Lambda R^{2}\right)
$$

Since this is a simple advection problem, the analytical solution is known at any time step by replacing $t$ by $U_{\infty}\left(x-x_{0}\right)$ with $U_{\infty}=0.5 c_{\infty}$. The time and space derivatives of $\mathbf{q}_{\text {in }}^{\prime}$ can thus be specified exactly in Eq. (2) (see Ref. [1]). The 2D compressible Euler equations are solved on a regular $101 \times 101$ grid. The results are depicted in Fig. 1 . The first test is made without updating the pressure fluctuations. The results in the first column of Fig. 1(a) indicate that the vorticity is almost perfectly entered in the box, but a monopolar spurious wave is emitted with an amplitude greater than $10 \mathrm{~Pa}$. When the pressure is correctly initialized in the three other cases in Figs. 1(b)-1(d), the pressure variations are depicted between -1 and $1 \mathrm{~Pa}$. The spurious noise is thus more than one order of magnitude lower. We now consider some implementation issues for the inflow boundary condition. On one hand, we have tried to implement the inflow condition for the first planes with indices $i=1$ to 3 or for $i=1$ to 5 . On the other hand, the variables $\left(\rho_{\text {in }}, \mathbf{u}_{\text {in }}, p_{\text {in }}\right)$ are updated at every physical time step, or at every substep of the Runge-Kutta scheme. The results for the three cases combining the two previous choices are depicted in Figs. 1(b)-1(d). The second column [Fig. 1(b)] indicates the existence of an almost dipolar spurious wave of $1 \mathrm{~Pa}$ when the condition is coded on three rows of points and updated every substep. Similar levels of spurious noise are obtained when five points are used to implement the condition with an update every time step. Finally, when using five points and an update every substep of the Runge-Kutta in the last column [Fig. 1(d)], the 

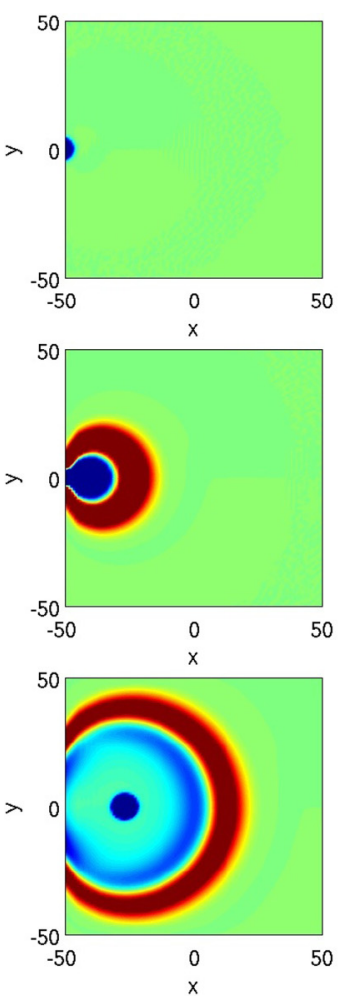

(a)
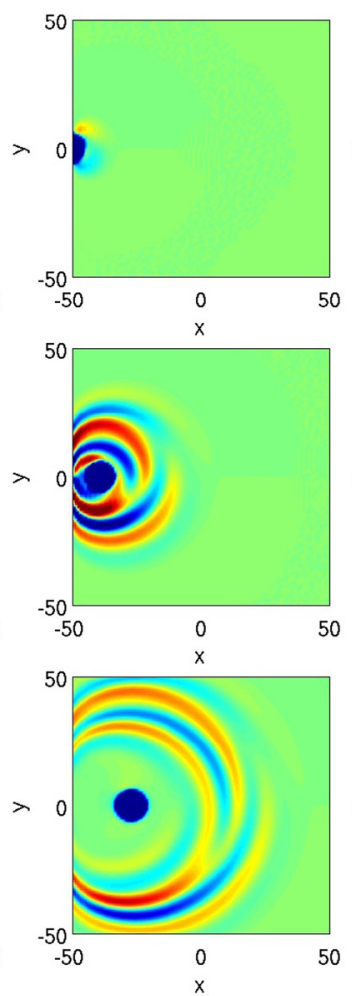

(b)
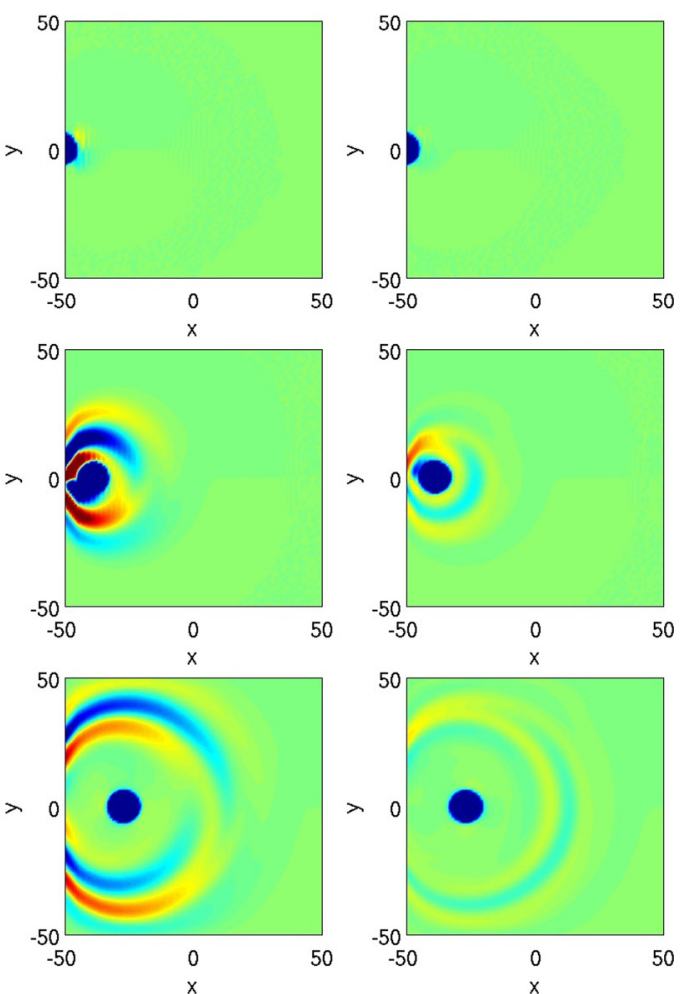

(c)

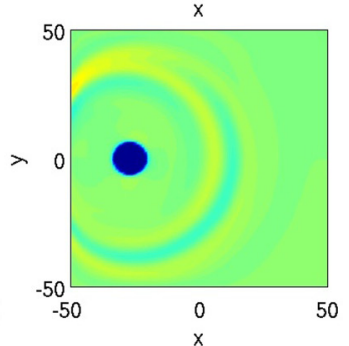

(d)

FIG. 1. Spurious fluctuating pressure due to the introduction of a vortex. Three successive instants are shown by using the inflow condition (2) for (a) $u^{\prime}, v^{\prime}$ only (levels $\pm 10 \mathrm{~Pa}$ ); (b) $u^{\prime}, v^{\prime}, p^{\prime}$ on three points and update every Runge-Kutta substep (levels $\pm 1 \mathrm{~Pa}$ ); (c) $u^{\prime}, v^{\prime}, p^{\prime}$ on five points and update every physical time step (levels $\pm 1 \mathrm{~Pa}$ ); (d) $u^{\prime}, v^{\prime}, p^{\prime}$ on five points and update every Runge-Kutta substep (levels $\pm 1 \mathrm{~Pa}$ ).

spurious noise can be further reduced. This last condition for the first five inlet planes is retained in the following, which is consistent with the eleven-point stencil of the finite-difference scheme.

In summary, at the upstream and upper boundaries of the computational domain, the radiation boundary conditions of Tam and Dong are applied on the five first rows parallel to the boundary. At the inlet, a triple decomposition is used to superimpose disturbances for all the state variables. The radiation center is on the wall and close to the inlet ( 30 points after) to minimize numerical artifacts due to the connection with the wall condition. At the outlet, the outflow boundary conditions of Tam and Dong [18], where the radiation solution is modified to allow the exit of vortical and entropic disturbances, is combined with a sponge zone dissipating vortical structures. This sponge zone uses grid stretching and a progressively applied Laplacian filter.

\section{CRITICAL OVERVIEW OF INFLOW TURBULENCE METHODS FOR AEROACOUSTICS}

In the light of the previous results, an overview of the numerous methods proposed to prescribe a turbulent inflow condition is proposed, focusing on the spurious noise sources induced by their numerical implementations. Several reviews have been proposed in the past [20-22] and show different strategies to reach rapidly a realistic turbulent state. The problematic is also common to interfaces between LES and Reynolds-averaged-Navier-Stokes (RANS) simulations. It is now well recognized that tripping conditions in both experiments and simulations can have a strong impact on the quality of the turbulence $[23,24]$. This is a sensitive issue for the development of incompressible 
wall turbulence. This is even more tricky when noise computations based on compressible equations are under investigation. Several tests have been conducted with more or less success in our team $[1-3,10,25]$ and are used to identify lessons learned and to point to new directions. For that purpose, the turbulent inflow methods are classified as synthetic turbulence methods, recycling techniques, and transition by modal interaction.

\section{A. Synthetic turbulence methods}

The simplest procedure is to superimpose random fluctuations on a desired turbulent mean velocity profile. But the development toward realistic turbulence is then lengthy. Improvements consist in constraining the amplitude of the fluctuations to satisfy a set of second-order statistics (i.e., Reynolds stress tensor) or a prescribed energy spectrum [26]. Lund et al. [27] proposed for example (in their Appendix A) a simple method to generate random fluctuations satisfying prescribed mean flow profiles and stress tensor. This method is based on random numbers decorrelated in both space and time. A spectral representation of the fluctuations can be used to retrieve certain correlations. For instance, Lee et al. [26] and Le et al. [28] computed random fluctuations compatible with a prescribed power spectrum and a random phase. This is a kind of inverse Fourier transform of turbulence spectra. The definition of the phase is crucial to ensure a sufficient randomization. $\mathrm{Na}$ and Moin [29] and Chung and Sung [30] constructed the fluctuations by the randomization of a frozen turbulent realization from DNS. A phase jittering technique was combined with Taylor's hypothesis in order to reach an unsteady turbulent state.

Another way to achieve rapidly a turbulent state is to impose fluctuations, bearing strong similarities with a turbulence field. This is sometimes called synthetic turbulence. The random Fourier modes (RFM), introduced by Kraichnan [31], are a good candidate. They were used, for example, for the prescription of a turbulent boundary layer ahead of a rectangular cavity [25]. The RFM are used to generate homogeneous turbulence with a prescribed energy spectrum. The way to unfreeze this turbulent field can be inspired by the stochastic noise generation and radiation (SNGR) formulation [32]. The original version, restricted to the generation of isotropic turbulence, was simply extended to inhomogeneous wall turbulence by convolving the modes with prescribed mean velocity profile and turbulent intensities [25]. This latter procedure violates the incompressibility constraint and therefore generates spurious acoustic waves. To cope with this, Smirnov et al. [33] applied a geometric transformation based on the Reynolds tensor matrix to the homogeneous synthetic turbulence. If the turbulence is initially divergence free, this property is preserved by the transformation [34]. Nevertheless only the velocity components are known, so that the adaptation of the inflow pressure still generate spurious waves, as demonstrated in Ref. [1], where different parametrizations of the RFM have been tested for triggering turbulent boundary layers. Another degree of sophistication was reached in the strategy of Sandham et al. [35], in which inner- and outer-layer disturbances with associated phase information are introduced. The inner disturbances represent lifted streaks, while the outer region comprises three-dimensional vortices. This method was applied for example to feed the turbulent boundary layer on one side of a thin plate to investigate trailing edge noise [36]. To reduce the spurious noise emitted by the inflow condition, a buffer layer was added near the inlet at the price of perturbing the transition toward developed turbulence. Another adaptation of this method was proposed to trigger a turbulent state ahead of a cylindrical cavity by Marsden et al. [37]. The idea to use vortical structures with prescribed length and time scales and a given shape was also developed by Jarrin et al. [38] to feed large eddy simulations of turbulent channel flows. Their synthetic eddy method (SEM) was improved by Pamiès et al. [39] for triggering turbulent boundary layers. A comparison between RFM and SEM methods was conducted for the development of a supersonic boundary layer [2] or for prescribing a turbulent boundary layer ahead of a cylindrical cavity [3]. It was concluded that SEM was more efficient than RFM to reach a turbulent state rapidly for a well-chosen set of parameters. However, the results clearly manifested the well-known memory effect, especially starting with a Reynolds number for which the flow should be initially turbulent. This conclusion was nicely shown in the study of Schlatter and Örlü [24], who tested the effects 
of different tripping conditions. The other conclusion in Refs. [2,3] was that the inflow conditions based on synthetic turbulence were noisy since a high level of forcing was required and, even if the synthetic velocity field is solenoidal, it was not possible to compute the associated thermodynamic quantities and the spurious noise was directly proportional to the amplitude of the dynamic pressure associated with inlet disturbances. One solution would be to reduce the level of perturbations, but the distance to reach a realistic turbulent state is then very long. Other synthetic methods have been proposed to reduce the artificial transitory phase but they suffer from the same limitations. We can quote the digital-filter-based approach proposed by Klein et al. [40], improved in several contributions [41-43], or the direct introduction of coherent structures such as hairpin-like vortices following the attached eddy hypothesis $[22,44]$.

\section{B. Recycling techniques}

Another class of methods relies on the idea of recycling the turbulence from another simulation or from the same simulation. The simplest examples are the temporal simulations, which rely on the homogeneity of the flow in the streamwise direction. Most of the earlier computations of turbulent boundary layers have used temporal simulations based on the quasiperiodicity of the flow in the streamwise direction. An extension was proposed by Spalart [45] which includes "growth terms" to rescale the outflow to the inflow. The idea was to define a set of coordinate lines along which the streamwise inhomogeneity associated with the boundary layer growth is minimized. The fringe method used by Flohr and Balaras [46] bears some similarities. Lund et al. [27] developed a more versatile method based on an auxiliary simulation to initiate a recycling and rescaling procedure, which is very popular. Numerous variants have been tested to overcome certain difficulties such as the initialization procedure, the decay of turbulence, or the introduction of a periodic component based on the distance between the inlet and the recycling plane. Concerning the latter issue, this long-range temporal periodicity of the inflow signal can trigger the unsteady behavior of a cavity flow or can generate spurious low-frequency noise by being diffracted by the trailing edge of an airfoil, for example. Liu and Pletcher [47] proposed slightly different conditions and suggested locating dynamically the recycling plane in order to reduce the startup transient and maintain a relatively short inlet section. Ferrante and Elghobashi [48] added a set of steps preceding the rescaling process, for ensuring the compatibility with statistical correlations and turbulent kinetic energy spectra, in order to sustain the production of turbulence. A more flexible approach coupled with a multiscale method was proposed by Araya et al. [49] to reach high Reynolds number or pressure-gradient conditions. The rescaling approach was extended in the compressible regime by several authors [50-52], who treated differently the thermodynamic variables. These improvements are necessary for supersonic conditions, where compressibility effects are important, but the approximate rescaling and recycling of thermodynamic quantities is not sufficient for aeroacoustic simulations, as shown for a cavity flow [3]. The reintroduced pressure disturbances do not ensure that the inlet perturbations are solutions of the compressible equations and an adaption phase is necessary and produces spurious noise. Moreover, acoustic waves present in the recycling plane are also reintroduced as spurious noise. Another original idea to achieve rapidly a turbulent state has been proposed by Spille and Kaltenbach [53]. It consists of using random numbers and introducing a body force in the framework of close-loop control to accelerate the adjustment process toward equilibrium turbulence and to control integral properties of the boundary layer. This idea was pursued by Laraufie et al. [54]. The recycling tricks are not limited to auxiliary simulations, and experimental quantities can be introduced in numerical simulations. Druault et al. [55] or Perret et al. [56] propose to combine proper orthogonal decomposition (POD) and linear stochastic estimation to establish an interface between experimental and numerical data. When using an auxiliary simulation, the transfer to the main simulation must occur at each time step and can therefore become prohibitively expensive. Recycling tricks have been proposed by $\mathrm{Na}$ and Moin [29] and by Xiong et al. [57] to reuse a turbulent entry without generating an artificial bias. Another promising idea is to construct a reduced order model of the wall turbulence dynamics [58]. The dynamical system resulting, for instance, from the projection on a POD basis, 
can described the coherent structure with a correct behavior in space and time. The high-frequency incoherent motions can then be reconstructed from random modes [58]. This idea was tested for generating turbulent inflow for a compressible boundary layer [1]. A compressible POD-Galerkin model was derived from an auxiliary turbulent boundary-layer simulation. This surrogate model was able to achieve a rapid development toward realistic turbulence, but suffered from a certain lack of robustness for long-term tracking [1].

\section{Transition by modal interaction}

Another simple idea to generate wall turbulence is to reproduce a physical transition scenario. An evident advantage is that very small perturbations are entered, reducing the spurious noise, which is generally proportional to the amplitude of the dynamic pressure associated with the velocity perturbations. The main drawback is that a simulation of the complete transition would require an expensive lengthening of the computational domain. The laminar/turbulent transition in a boundary layer can follow many possible routes [59]. The traditional scenario arises from the exponential growth of primary instabilities, e.g., Tollmien-Schlichting (TS) waves for a Blasius boundary layer, together with subsequent secondary instabilities. The $K$-type transition discovered by Klebanoff et al. [60] or the subharmonic transition described by Kachanov et al. [61] ( $N$-type) and Herbert [62] ( $H$-type) belong to this category. A popular technique to reproduce these scenarios with incompressible flow solvers is the use of a numerical blowing and suction condition at the wall. For instance, this kind of forcing was used by Rist and Fasel [63] to reproduce the vibrating ribbons used in the experiments on boundary-layer transition. The parametrization of this numerical excitation was discussed by Berlin et al. [64] and Schlatter and Örlü [24]. In order to shorten the transition length, it can be beneficial to bypass the role of the primary instability mechanism. Schmid and Henningson [59] describe this new route to turbulence as emanating from nonmodal growth mechanisms. The receptivity to freestream turbulence (FST) is one possible bypass scenario which has been reproduced in numerical simulations. Nagarajan et al. [65] and Ovchinnikov et al. [66] showed how the vortical disturbances from freestream turbulence can penetrate inside the boundary layer near a leading edge. Jacobs and Durbin [67] reproduced the bypass transition due to freestream disturbances without mediation by a leading edge. Their freestream perturbations consist in continuous Orr-Sommerfeld (OS) and Squire (SQ) modes, which are oscillatory in the free stream and decay inside the boundary layer. In all FST scenarios, low-frequency disturbances generate breathing modes inside the boundary layer, called Klebanoff modes. Long streaks of streamwise velocity perturbation are initiated and seem remarkably stable. Their liftup from the wall results in an inflectional velocity profile, which becomes prone to short-wavelength instabilities of the Kelvin-Helmholtz type triggered by the high-frequency FST. These instabilities intensify downstream and burst into turbulent spots. The route to turbulence is similar for the streak breakdown, where a secondary instability of streaks causes breakdown to turbulence [68]. Three-dimensionality plays a key role and the elongated streamwise structures are generated by a transient growth mechanism. Optimal disturbances relative to this transient growth take besides the form of streamwise streaks for a Blasius profile [69] and a Falkner-Skan profile [70]. Similar conclusions are obtained for a compressible boundary-layer flow [71]. Since these perturbations experience the maximum spatial energy growth in the linear regime, they are interesting candidates to simulate a rapid transition. As FST scenarios, the breakdown starts with a local rapid growth of the normal velocity bringing low-speed fluid from the wall (liftup mechanism). Soon hereafter a turbulent spot is formed [72]. This phenomenon is similar to the low-velocity spikes previously observed in experiments for the late stages of $K$-type or $N$-type transitions [73,74]. Common features of all scenarios are the formation of an inflectional velocity profile before the turbulent breakdown and the important role of modal interactions and three-dimensionalities. Zaki and Durbin [75] showed, for instance, that the Klebanoff mode in FST results from a coupling between continuous spectrum OS and SQ modes. The combination of one strongly coupled and one weakly coupled high-frequency mode is able to induce a complete transition process. As discussed in detail by Yaglom [76], modal interaction is also a key ingredient 
in the oblique transition scenario ( $O$-type) $[64,77]$ and the triad scenario ( $C$-type) of Craik [78]. In the compressible regime, oblique TS waves are often present, and streamwise streaks are also at work in FST transition [79]. A laminar separation bubble can also induce a rapid transition. If two-dimensional disturbances are introduced, large two-dimensional vortices are shed and the breakdown then shows similarities to the one due to secondary instabilities in mixing layers. Rist and Maucher [80] have shown that if oblique waves are introduced, no large two-dimensional vortices are formed; instead the flow quickly becomes turbulent. This nonexhaustive panorama shows that several ingredients such as inflectional velocity profiles due to late stages of transition or to separation, three-dimensional perturbations in the form of oblique waves or peak and valley modulations, and streamwise vortices can be used to favor a rapid transition.

\section{PROPOSED INFLOW BOUNDARY CONDITION}

\section{A. Numerical strategy}

The observations made in Secs. II and III guided the choices to construct a silent inflow condition to access acoustic emissions from a turbulent boundary layer, which is a poorly efficient radiator. Flow disturbances, including the associated thermodynamic quantities, will be introduced in the computational domain using the Tam and Dong inflow condition. Since it is difficult to specify correctly the pressure and density fluctuations in synthetic turbulence or recycling methods, we choose to use a transition by modal interaction. To reduce issues remaining in the implementation of the numerical boundary conditions, the amplitude of disturbances should be as low as possible. Considering that the late stages of transition imply a local inflectional velocity profile prone to Kelvin-Helmholtz $(\mathrm{KH})$ instabilities with an amplification rate one order of magnitude greater than a viscous instability such as TS waves, it was decided to start directly from an inflectional velocity profile. A triad of unstable modes, comprising a pair of oblique modes and a two-dimensional mode, is selected using the linearized compressible Navier-Stokes equations (Appendix A), so that the pressure and density perturbations are known at the inlet.

Since very few numerical studies have considered oblique-wave transition and are furthermore based on spectral solvers for the incompressible equations, a first validation step is to check the ability of our compressible finite-difference code to reproduce an $O$-type transition. The oblique-wave transition of the Blasius flow studied by Joslin et al. [81] is reproduced at a low Mach number, $M=0.1$. The results, presented in Appendix B, are in fairly good agreement with both DNS and parabolic stability equations (PSE) theory. In particular, the amplification of the streaks is well reproduced and can lead to the eruption of turbulence through a liftup mechanism if the initial amplitude is sufficiently high.

\section{B. Summary of the proposed inflow strategy}

The proposed procedure can be summarized by the following steps:

(1) 2D computation of the steady flow behind a backward-facing step of height $h$ (representative of the end of a triggering strip) with the same solver and the same grid as the 3D computation (convergence of residuals in roughly 300000 iterations);

(2) Convergence $(\sim 200000$ iterations) in $2 \mathrm{D}$ without the step (initialized with the previous computation 7 points after the end of the step, corresponding to $0.49 h$ ) to ensure a proper adaptation of the nonreflecting condition at inlet;

(3) The obtained base flow is used for a local stability analysis of compressible linearized Navier-Stokes equations in the inlet plane;

(4) The computation is restarted in 3D from the base flow with a triad of unstable KelvinHelmholtz-type modes with low amplitude. The Tam and Dong boundary condition is used to impose the inflow quantities, including pressure disturbances.

The 3D grid without the step has $n_{x}=1440, n_{y}=400, n_{z}=400$ ( $\sim 320$ million points). The flow conditions are the same as the previous studies on boundary layer noise $[4,7,8]$. In particular, 


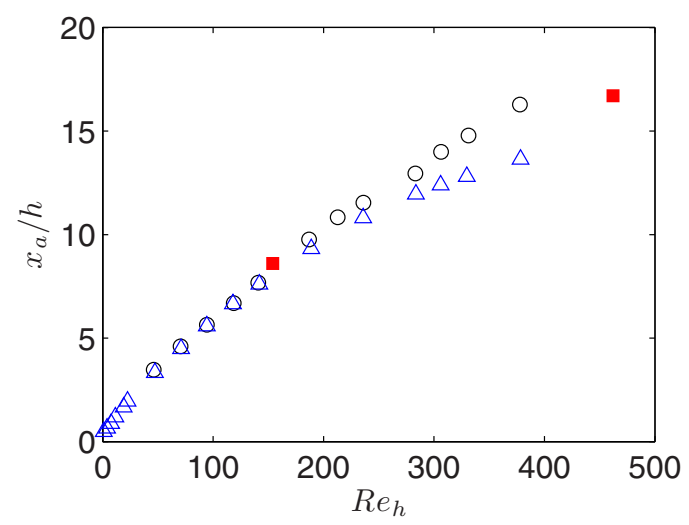

FIG. 2. Attachment length $x_{a}$ for the 2D base flow $(\square)$ compared to channel flow experiments (step height $h=0.9423$ channel height) by Armaly et al. [82] (००) and incompressible channel flow simulations (same conditions) by Biswas et al. [83] $(\triangle \triangle)$.

the Mach number is 0.5 . Note also that the resolution is the $x$ and $y$ directions are the same as the previous LES but the spanwise domain has been considerably enlarged and the mesh size in this direction is refined to roughly 15 (in wall units). Knowing the spanwise periodicity of the 3D instability waves, as discussed later, we have also chosen a spanwise extent which is a multiple of the spanwise wavelength $\lambda_{z}=2 \pi / \beta$, where $\beta$ is the spanwise wave number of disturbances, which can be related to the wave angle. Here we have $L_{z}=8 \lambda_{z}$.

\section{Base flow behind a backward-facing step}

First, a base flow is obtained from the solution of the 2D Navier-Stokes equations without inlet perturbations. The 2D computational domain is a slice of the 3D mesh with an upstream extension to include a backward-facing step. The number of points in the $x$ direction is now 1504, whereas $n_{y}$ is unchanged. The size of the step is 58 points in $x$ and 18 points in the $y$ direction. The step height is $h \simeq 4 \times 10^{-5} \mathrm{~m}$ and the Mach number is fixed at 0.5 , yielding a Reynolds number $\operatorname{Re}_{h}=462$. Note that the step height $h$ and the freestream velocity $U_{\infty}=M c_{\infty}$ are used in the following to make the quantities nondimensional. The initial Blasius boundary layer above the step has a thickness $\delta_{\text {in }}=2.32 h$.

In the absence of inlet perturbations, a steady solution can be reached due to the convective nature of the instabilities. After a transient period, the flow is seen to relax toward a steady state, 300000 iterations being necessary to saturate residual values. The backward-facing step flow is validated against experimental and numerical results in Fig. 2. Since the present Reynolds number is above the range studied in Refs. [82,83], another computation at $\mathrm{Re}_{h}=154$ has been performed. The attachment length $x_{a}$ is in fair agreement with previous works. For the higher Reynolds number of the step (with a height representative of experimental trip bands), the attachment length is also compatible with an extrapolation of the data.

Then, a subdomain is extracted by excluding the step. The new inlet is seven points behind the step, i.e., $0.49 h$. Before running the 3D code, the base flow is run 200000 more iterations on the 2D truncated domain to ensure a good adaptation of the inflow boundary. The mean flow profiles, which will be used for the stability calculations, are depicted in Fig. 3.

\section{Determination of a resonant triad}

The base flow is used for a local stability analysis using the linearized compressible Navier-Stokes equations. Details about the stability solver are given in Appendix A. Three-dimensional spatial 

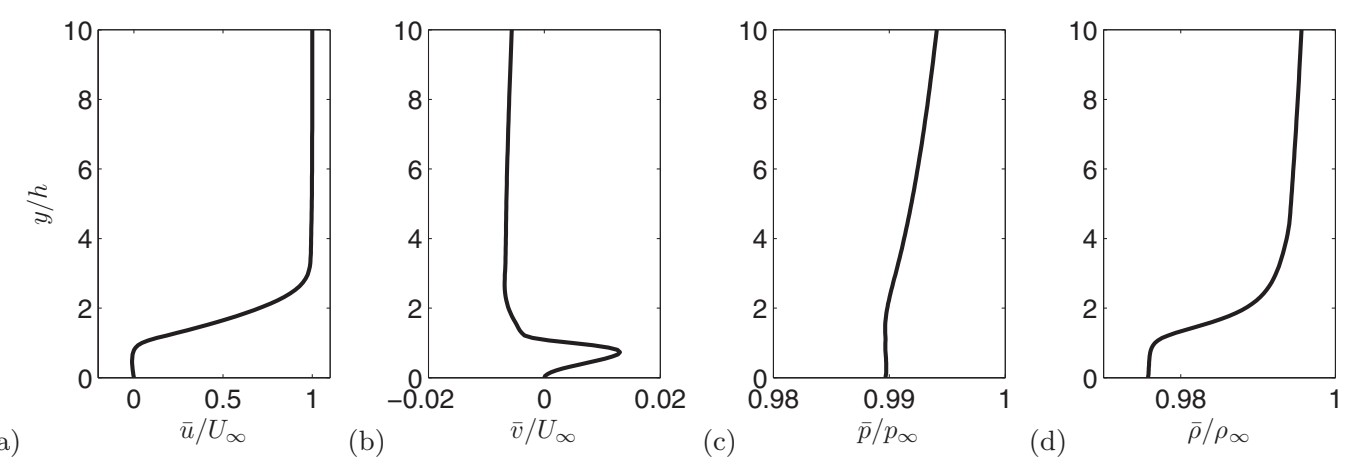

FIG. 3. Base flow obtained seven points downstream of the step at $M=0.5, \operatorname{Re}_{h}=462$. From left to right, streamwise velocity, vertical velocity, pressure, and density.

modes are searched with a real angular frequency $\omega$ and a complex wave number $\mathbf{k}=\alpha \mathbf{e}_{x}+\beta \mathbf{e}_{z}$. The streamwise component of the wave number is a complex number, $\alpha=\alpha_{r}+i \alpha_{i}$, where $\alpha_{i}$ thus represents the amplification factor. The spanwise component $\beta$ defines the wave angle $\Psi=$ $\arctan \left(\beta / \alpha_{r}\right)$.

The possibility to use resonant modes was first discussed by Kelly [84] in the context of shear flows. He showed that in addition to the exchange of energy among the wave components owing to their interaction, a transfer of energy from the base flow to the disturbance may also occur. This idea was used by Craik [78] to study the transition of boundary-layer flow. He selected a triad of Tollmien-Schlichting (TS) waves such that

$$
\omega_{1}+\omega_{2}=\omega_{3} \quad \text { and } \quad \beta_{1}+\beta_{2}=\beta_{3} \quad \text { and } \quad \alpha_{r 1}+\alpha_{r 2}=\alpha_{r 3} .
$$

If the triplet is symmetric, the following relationships hold:

$$
\omega_{1}=\omega_{2}=\omega_{3} / 2 \text { and } \beta_{1}=-\beta_{2} ; \beta_{3}=0 \text { and } \alpha_{r 1 / 2}+\alpha_{r 1 / 2}=\alpha_{r 1} .
$$

The values with subscript 3 represent a two-dimensional wave, whereas the two other waves are oblique at equal and opposite angles to the flow direction that are subharmonic of the previous one. We thus note with subscript $\bullet_{1}$ and $\bullet_{1 / 2}$ the 2D fundamental and the 3D subharmonics, respectively. From (6), the phase velocities, $c_{\varphi}=\omega / \alpha_{r}$ of the waves are equal, i.e., $c_{\varphi 1 / 2}=c_{\varphi 1}$.

Transition with a triad of resonant TS waves has been shown to be a subclass of the $H$-type scenario [76]. In the first experiments about the $H$-type transition, Kachanov and Levchenko [61] excited the 2D fundamental and oblique subharmonics but the triad was not necessarily resonant. Herbert [62] has explained theoretically this scenario with secondary instabilities by using Floquet's theory about a basic state consisting of a Blasius flow and 2D TS waves. The link between the subharmonic $H$-type and the resonant-triad ( $C$-type after Craik) scenarios has clearly been made by Zelman and Maslennikova [85], using weakly nonlinear stability theory (see the review by Yaglom [76]). Besides, experiments with resonant triads have been carried out by Corke and Mangano [86] or Borodulin et al. [87,88]. They also studied detuned triads where the phase condition is not fulfilled, even in antiphase [87,89]. Note that the experiments of Borodulin et al. $[87,88]$ considered the transition of a Falkner-Skan flow with a mild adverse pressure gradient. The introduction of their first paper [87] gives a comprehensive overview of subharmonic transition. As fate would have it, Craik was investigating the resonant triad to get some insight into the experiments of Klebanoff et al. [60], which pertains to the other scenario ( $K$ type). Nevertheless, Bake et al. [74] have shown that there are in fact similarities between both scenarios, notably in the late stages of transition. For instance, the presence of $\Lambda$-shape structures is common to both cases, but they appear aligned in the $K$-type transition whereas they are staggered with respect to the spanwise direction in the $H$-type transition. Furthermore, the generation of vortex rings and spikes at the late evolution of 


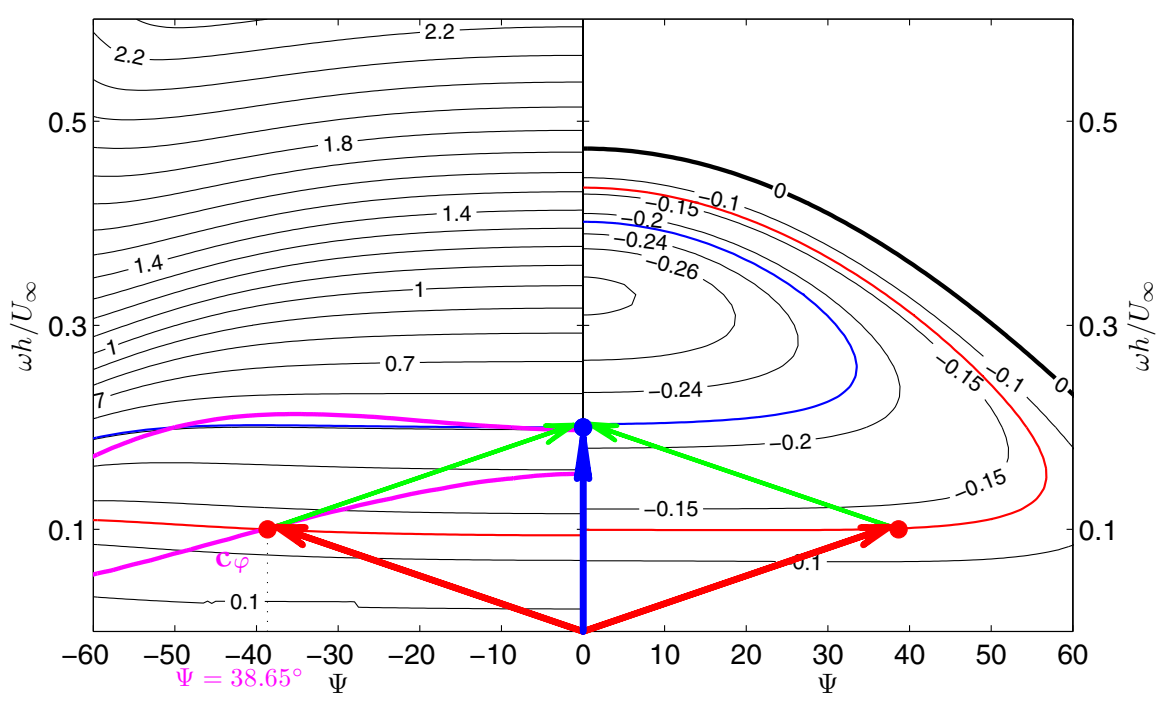

FIG. 4. Choice of a Craik's triad: on the left, dispersion curves for the wave number $\alpha_{r}$ as function of negative wave angle $\Psi$, and on the right, amplification factor $\alpha_{i}$ as function of positive wave angle $\Psi$. Red and blue isolines are associated to the 2D fundamental wave $(\mathbf{)}$ ) and $2 \mathrm{D}$ fundamental wave ( ), respectively. The magenta isoline in the left part represents the phase velocity, identical for the three waves.

the $\Lambda$ vortices can also be a common feature. Another similarity is the importance of the three dimensionality with peaks and valleys, and the presence of an inflection point in the instantaneous streamwise velocity profiles prior to the turbulent breakdown.

In the present study, as in the experiments of Corke and Mangano [86], a Craik's triad is considered with equal amplitude for the fundamental and subharmonic waves. However, as shown previously in Sec. IV C, the base flow is not the Blasius solution but it has an inflectional profile and a triad of Kelvin-Helmholtz-type (KH) modes is selected, in the same manner as done by Kelly [84]. The stability code, presented in Appendix A, is used to provide a map of dispersion curves shown in Fig. 4. Note that the quantities are symmetrical about the $\Psi$ axis, so half maps are plotted. The neutral stability curve is marked by the thick line in the right part of Fig. 4. A large range of frequencies are unstable with a maximum growth rate, $-\alpha_{i}$, for $\omega h / U_{\infty} \approx 0.33$. This nondimensional value for the most unstable wave is higher than the one obtained for a boundary-layer velocity profile, which is close to 0.1 using the edge velocity and displacement thickness as velocity and length scales respectively. That is why a lower frequency $\omega h / U_{\infty}=0.2$ has been arbitrarily chosen for the fundamental wave, yielding a frequency of 0.1 for the subharmonics. The selected values are of the same order as the dimensionless values used by Craik [78]. The oblique-wave frequency is also close to that used in Appendix B for the Blasius flow, based on the initial displacement thickness [which is between $h$ and $2 h$ in the present LES as shown later, e.g., in Fig. 9(a)]. Note that the growth rate is of the order of -0.1 for the $\mathrm{KH}$ oblique waves, more than one order of magnitude greater than the value -0.002 found in Appendix B for the Blasius flow. This illustrates the advantage of using a velocity profile with an inflection point. For instance, a disturbance amplitude of $2 \times 10^{-2}$ is necessary in the case of Blasius flow of Appendix B to achieve a transition length comparable to that obtained with the inflectional base flow with a disturbance amplitude of $6 \times 10^{-4}$.

Once the frequency of the 2D fundamental is fixed ( $\omega_{1}$ and $\alpha_{r 1}$ known), the wave angle of the 3D subharmonic at $\omega_{1 / 2}=\omega_{1} / 2$ is determined by the intersection of the red isoline $\alpha_{r 1 / 2}=\alpha_{r 1} / 2$ and the magenta isoline $c_{\varphi 1 / 2}=c_{\varphi 1}$ in the left part of Fig. 4. A wave angle $\Psi$ of $38.6^{\circ}$ is obtained. From the local linear stability analysis, we get the following: 


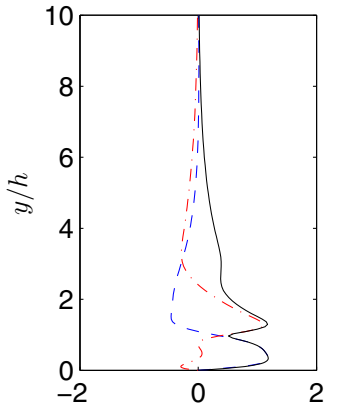

(a)

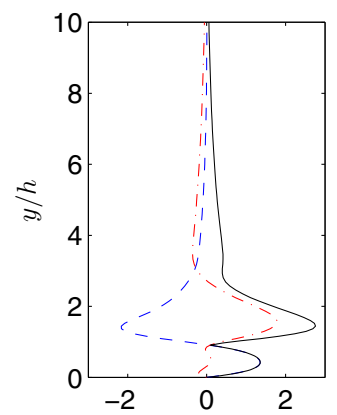

(e)

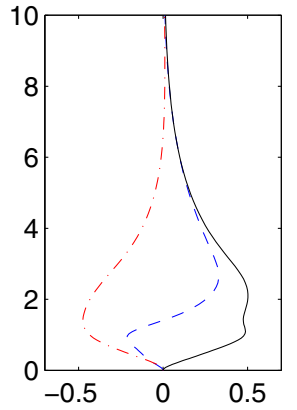

(b)

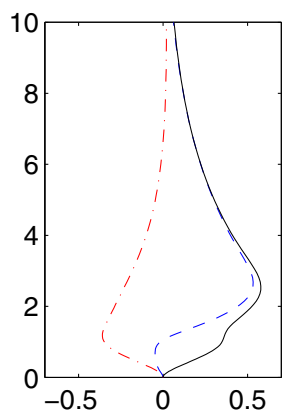

(f)

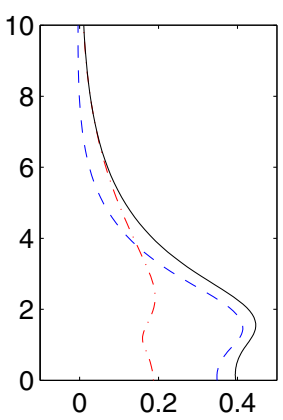

(c)

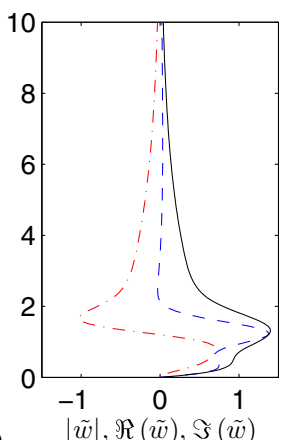

(g)

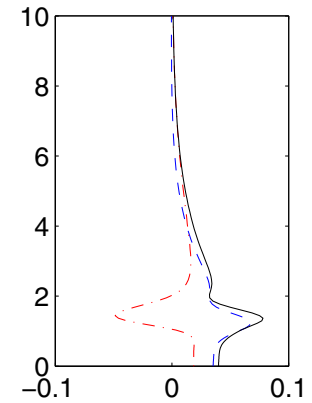

(d)

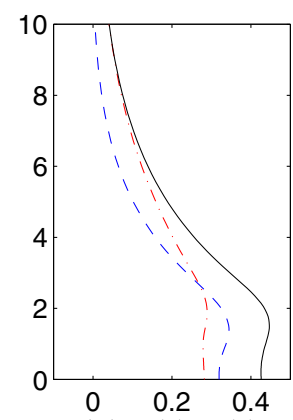

(h)

$|\tilde{p}|, \Re(\tilde{p}), \Im(\tilde{p})$

FIG. 5. Eigenfunctions from the local stability analysis for the 2D fundamental [(a)-(d)] and the 3D subharmonic [(e)-(h)]: modulus; _ _ _, real part; and _ . _ ., imaginary part.

(1) 2D fundamental Kelvin-Helmholtz-type mode:

$$
\omega_{1}=0.2 U_{\infty} / h, \alpha_{r 1}=0.5059 / h \text {, and } \alpha_{i 1}=-0.2179 / h \text {, }
$$

(2) 3D oblique modes:

$\omega_{1 / 2}=0.1 U_{\infty} / h, \alpha_{r 1 / 2}=0.2529 / h, \alpha_{i 1 / 2}=-0.1309 / h$, and $\beta_{1 / 2}= \pm \alpha_{r 1 / 2} \tan (38.65 \pi / 180)$.

The eigenfunctions $\tilde{\mathbf{q}}$ obtained for the modes forming the triad for the different primitive variables $\tilde{u}, \tilde{v}, \tilde{w}, \tilde{p}$, and $\tilde{T}$ are depicted in Fig. 5 . In this way, the pressure perturbations $p_{\text {in }}^{\prime}$ and the density perturbation $\rho_{\text {in }}^{\prime}$ (deduced from $p^{\prime}$ and $T^{\prime}$ using ideal gas law), which are associated with the velocity disturbances, are known and can be imposed in the inlet boundary condition to avoid spurious noise as

$$
\begin{aligned}
\mathbf{q}_{\text {in }}^{\prime}(x, y, z, t)= & \varepsilon_{2 \mathrm{D}} \mathrm{e}^{-\alpha_{i 1} x}\left[\operatorname{Re}\left(\tilde{\mathbf{q}}_{1}(y)\right) \cos \left(\alpha_{r 1} x-\omega_{1} t\right)-\operatorname{Im}\left(\tilde{\mathbf{q}}_{1}(y)\right) \sin \left(\alpha_{r 1} x-\omega_{1} t\right)\right] \\
& +\varepsilon_{3 \mathrm{D}} \mathrm{e}^{-\alpha_{i 1 / 2} x}\left[\operatorname{Re}\left(\tilde{\mathbf{q}}_{1 / 2}(y)\right) \cos \left(\alpha_{r 1 / 2} x-\omega_{1 / 2} t+\beta_{1 / 2} z\right)-\operatorname{Im}\left(\tilde{\mathbf{q}}_{1 / 2}(y)\right) \sin \left(\alpha_{r 1 / 2} x\right.\right. \\
& \left.\left.-\omega_{1 / 2} t+\beta_{1 / 2} z\right)\right]+\varepsilon_{3 \mathrm{D}} \mathrm{e}^{-\alpha_{i 1 / 2} x}\left[\operatorname{Re}\left(\tilde{\mathbf{q}}_{1 / 2}(y)\right) \cos \left(\alpha_{r 1 / 2} x-\omega_{1 / 2} t-\beta_{1 / 2} z\right)\right. \\
& \left.-\operatorname{Im}\left(\tilde{\mathbf{q}}_{1 / 2}(y)\right) \sin \left(\alpha_{r 1 / 2} x-\omega_{1 / 2} t-\beta_{1 / 2} z\right)\right]
\end{aligned}
$$

where $\mathbf{q}_{\text {in }}^{\prime}=\left(\rho_{\text {in }}^{\prime}, \mathbf{u}_{\text {in }}^{\prime}, p_{\text {in }}^{\prime}\right)^{T}$.

Note that in the inflow boundary condition formulation, Eq. (2), the temporal $(\partial / \partial t)$ and spatial derivatives $(\partial / \partial r \equiv \cos \theta \partial / \partial x+\sin \theta \partial / \partial y)$ are required. Since the spatial and temporal dependences of modes are known, the time and spatial derivatives are computed analytically. For 
instance, $\partial \mathbf{q}^{\prime} / \partial t$ is given by

$$
\begin{aligned}
\frac{\partial \mathbf{q}_{\text {in }}^{\prime}(x, y, z, t)}{\partial t}= & \varepsilon_{2 \mathrm{D}} \omega_{1}\left[\operatorname{Re}\left(\tilde{\mathbf{q}}_{1}(y)\right) \sin \left(\alpha_{r 1} x-\omega_{1} t\right)+\operatorname{Im}\left(\tilde{\mathbf{q}}_{1}(y)\right) \cos \left(\alpha_{r 1} x-\omega_{1} t\right)\right] e^{-\alpha_{i 1} x} \\
& +\varepsilon_{3 \mathrm{D}} \omega_{1 / 2}\left[\operatorname{Re}\left(\tilde{\mathbf{q}}_{1 / 2}(y)\right) \sin \left(\alpha_{r 1 / 2} x-\omega_{1 / 2} t+\beta_{1 / 2} z\right)+\operatorname{Im}\left(\tilde{\mathbf{q}}_{1 / 2}(y)\right) \cos \left(\alpha_{r 1 / 2} x\right.\right. \\
& \left.\left.-\omega_{1 / 2} t+\beta_{1 / 2} z\right)\right] e^{-\alpha_{i 1 / 2} x} \\
& +\varepsilon_{3 \mathrm{D}} \omega_{1 / 2}\left[\operatorname{Re}\left(\tilde{\mathbf{q}}_{1 / 2}(y)\right) \sin \left(\alpha_{r 1 / 2} x-\omega_{1 / 2} t-\beta_{1 / 2} z\right)+\operatorname{Im}\left(\tilde{\mathbf{q}}_{1 / 2}(y)\right) \cos \left(\alpha_{r 1 / 2} x\right.\right. \\
& \left.\left.-\omega_{1 / 2} t-\beta_{1 / 2} z\right)\right] e^{-\alpha_{i 1 / 2} x}
\end{aligned}
$$

\section{E. Selection of a forcing amplitude}

Since the stability analysis is linear, we need to prescribe an amplitude for the inlet perturbations, $\varepsilon_{2 \mathrm{D}}$ for the fundamental and $\varepsilon_{3 \mathrm{D}}$ for the subharmonics in Eq. (7). An equal amplitude for the fundamental and subharmonic waves, $\varepsilon_{2 \mathrm{D}}=\varepsilon_{3 \mathrm{D}}=\varepsilon$ is retained, as in the experiments of Corke and Mangano [86]. Different values in the range $10^{-4}$ to $10^{-3}$ have been tested on a subdomain of the full LES. The flow development is depicted by the top views of the vertical velocity in Figs. 6(a)-6(d). For values lower than $\varepsilon=4 \times 10^{-4}$, no turbulence breakdown is observed in the box. By increasing $\varepsilon$, the eruption of turbulence arises closer to the inlet. We finally selected in the following a value of $\varepsilon=6 \times 10^{-4}$, close to the lowest amplitude for breakdown, for two reasons: (i) The amplitude of spurious noise from the inlet is proportional to $\varepsilon$, so a lower value yields a weaker perturbation, and (ii) the turbulence breakdown is well separated from the inlet boundary, so it is easier to identify if an eventual spurious noise is coming from the inlet boundary condition or from the breakdown region. To illustrate the benefit of using a triad of modes, the case of oblique waves alone (i.e., $\varepsilon_{2 \mathrm{D}}=0$ ) has been tested. The results for $\varepsilon_{3 \mathrm{D}}=8 \times 10^{-4}$ and $1 \times 10^{-3}$ are visualized in Figs. 6(e)-6(f). For an amplitude of $8 \times 10^{-4}$, the transition occurred rapidly with a triad (for $x / h \simeq 200$ ), whereas the breakdown is not present in the computational box when only the subharmonic waves are introduced. The transition length observed for an amplitude of $1 \times 10^{-3}$ is close to that observed with the triad with an amplitude of $6 \times 10^{-4}$, meaning that the resonant triad promotes a quicker transition. Finally, the case where only the 2D wave is entered is depicted in Fig. $6(\mathrm{~g})$. Even with an amplitude of $1 \times 10^{-3}$, a fast decay of the instability waves is observed. The pictures of disturbance development in Figs. 6(a)-6(f) show a checkerboard pattern (for $x / h \lesssim 50$ ), due to the pair of oblique waves with opposite angles, followed by the eruption of $\Lambda$-shape structures (for $50 \lesssim x / h \lesssim 120$ ). A staggered arrangement characteristic of the $H$-type transition is visible. Then streaks develop with a double spanwise wavelength with respect to the wavelength of initial oblique waves. Depending on the disturbance amplitude, streaky vortices remain stable or experience a subsequent wavelength doubling or high-frequency oscillations near the edge of the streaks, which can rapidly lead to turbulence [64]. The transition does not lead to the appearance of turbulent spots and is rather characterized by a continuous spectral enrichment, as shown later. The scenario thus pertains to the class of subharmonic transition. The details of the transition process from the location where $\Lambda$-shaped structures emerge is reminiscent of the observations made for the oblique transition in numerical [64] or experimental [77] studies. The presence of an inflexion point in the velocity profile thus considerably shortens the distance required in the primary phase, and the transition follows thereafter boundary-layer physics.

A modal analysis has been carried out to investigate the role of the Fourier components in the transition process. The velocity field is stored on volumes, corresponding to a streamwise length of $550 h$ (450 uniformly spaced points), a height of $19 h$ (100 points), and spanwise width $62 h=2 \lambda_{z}$ (100 uniformly spaced points), during 120000 iterations every 30 time steps (4000 samples). A fast Fourier transform is applied in time and in the spanwise direction and the notation $(\omega, \beta)$ is used for the Fourier components, where $\omega$ and $\beta$ are the angular frequency and spanwise wave number normalized by $\omega_{1 / 2}$ and $\beta_{1 / 2}$ respectively. The initial triad is thus represented by the oblique waves $(1,1)$ and $(1,-1)$ and the $2 \mathrm{D}$ fundamental $(2,0)$. Different quantities are studied in the literature, such 


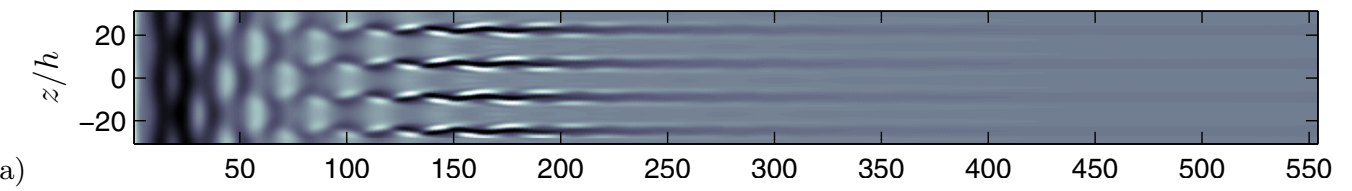

(a)

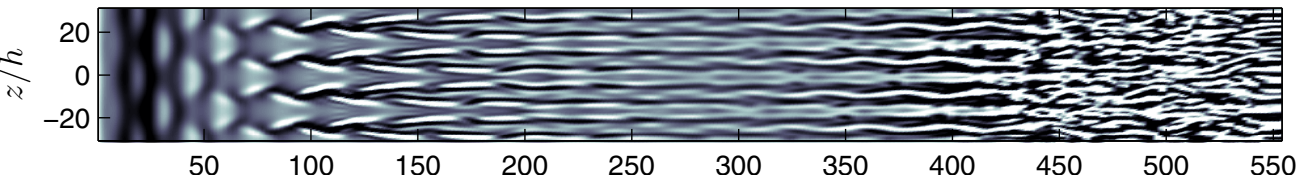

(b)

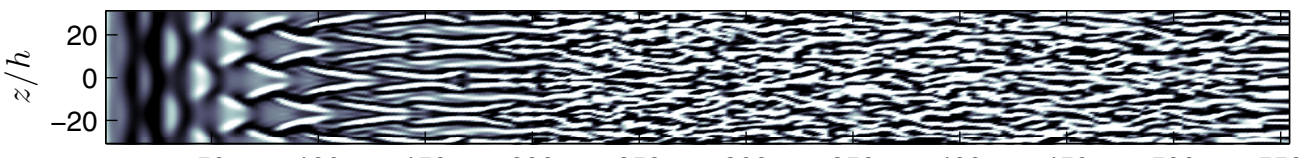

(c)
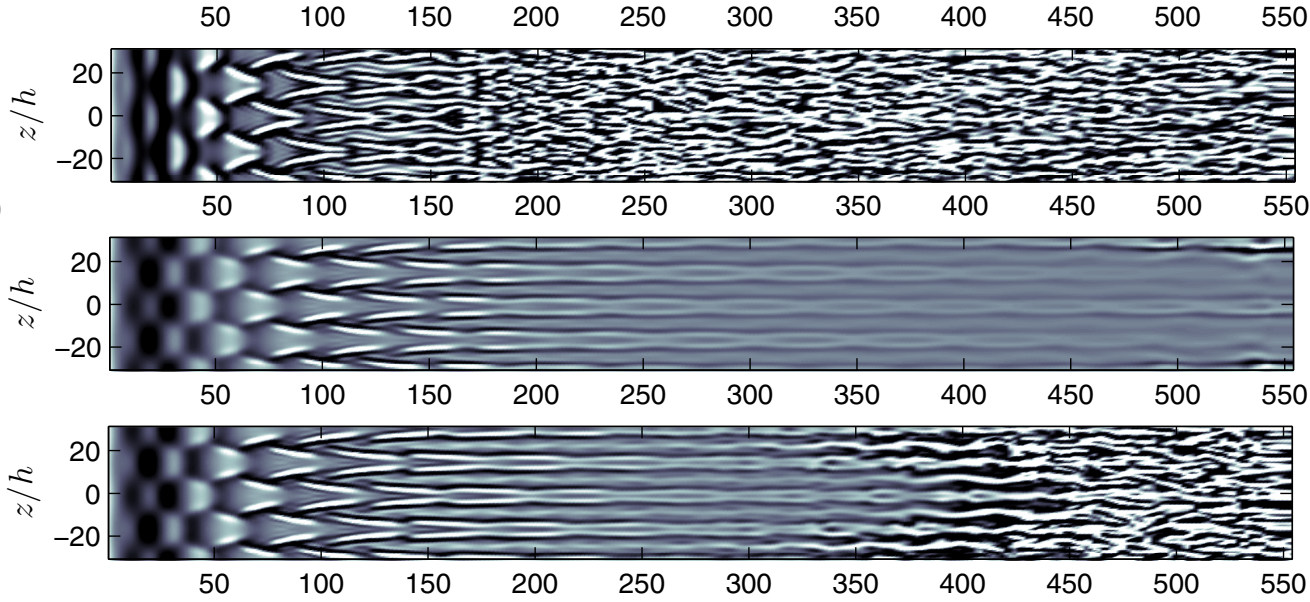

(f)

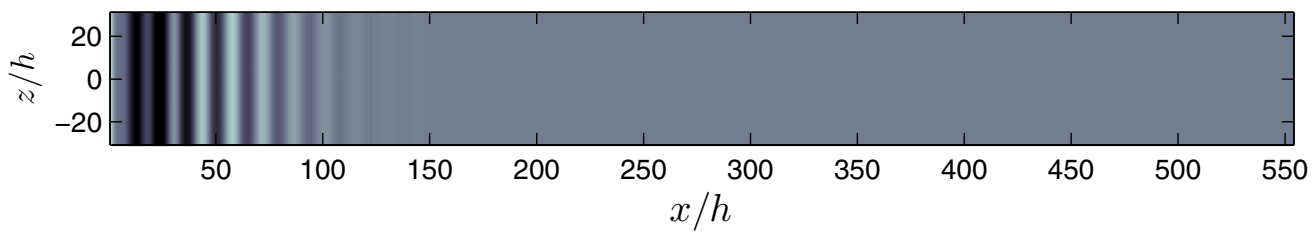

FIG. 6. Instantaneous views in the streamwise-spanwise plane at $y / h=0.61$ of the vertical velocity for different forcing amplitudes: (a) $\varepsilon_{2 \mathrm{D}}=\varepsilon_{3 \mathrm{D}}=4 \times 10^{-4}$; (b) $\varepsilon_{2 \mathrm{D}}=\varepsilon_{3 \mathrm{D}}=6 \times 10^{-4}$; (c) $\varepsilon_{2 \mathrm{D}}=\varepsilon_{3 \mathrm{D}}=8 \times 10^{-4}$; (d) $\varepsilon_{2 \mathrm{D}}=\varepsilon_{3 \mathrm{D}}=1 \times 10^{-3}$; (e) $\varepsilon_{2 \mathrm{D}}=0$ and $\varepsilon_{3 \mathrm{D}}=8 \times 10^{-4}$; (f) $\varepsilon_{2 \mathrm{D}}=0$ and $\varepsilon_{3 \mathrm{D}}=1 \times 10^{-3}$; (g) $\varepsilon_{2 \mathrm{D}}=1 \times 10^{-3}$ and $\varepsilon_{3 \mathrm{D}}=0$. Same color scale $\pm 1 \mathrm{~m} / \mathrm{s}$ for all figures.

as the maximum along $y$ of the modulus of the streamwise velocity component, $\max _{\{y\}}\left(\hat{u} \hat{u}^{*}\right)$, or the modal energy, which can be defined for a compressible fluid by taking into account both kinetic and internal energies:

$$
E_{m}=\int_{0}^{y_{\max }}\left[\frac{1}{2} \bar{\rho}\left(\hat{u}^{*} \hat{u}+\hat{v}^{*} \hat{v}+\hat{w}^{*} \hat{w}\right)+\frac{\hat{p}^{*} \hat{p}}{\bar{p}(\gamma-1)}\right] d y,
$$

where $\hat{\bullet}$ is used for Fourier components, $\boldsymbol{\bullet}$ for mean quantities, and $*$ denotes the complex conjugate. A separate study of the terms in Eq. (8) shows that the contribution of streamwise velocity perturbations overwhelms the other components, and that the contribution of internal energy is negligible for the subsonic speed under consideration. The study of $\hat{u} \hat{u}^{*}$ at the height where it is a maximum, which is notably used in experiments [77], yields the same modal evolutions as the 


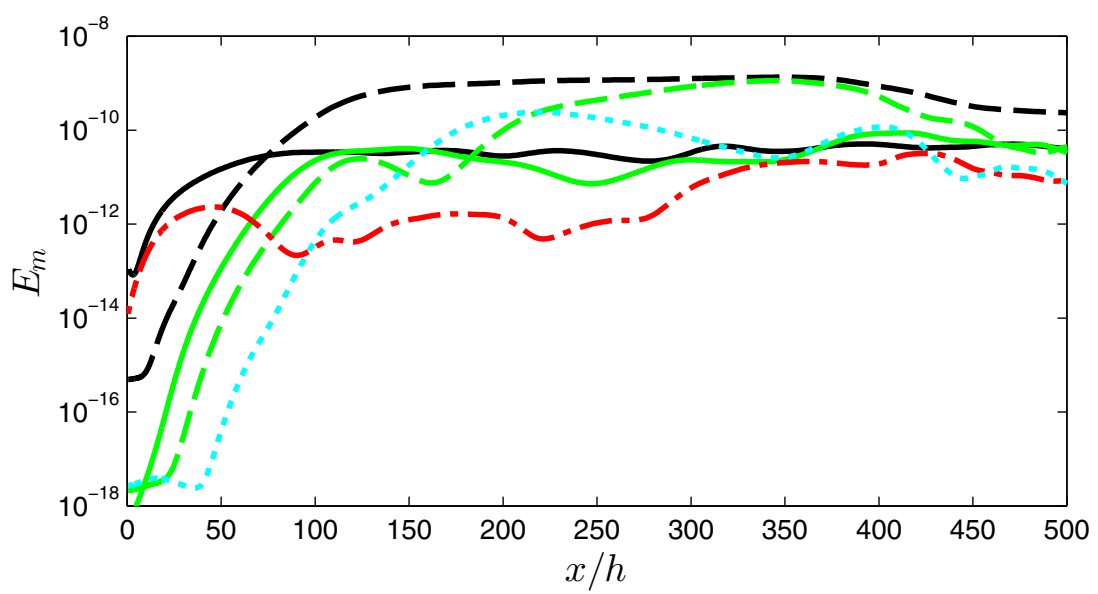

FIG. 7. Evolution of the integrated modal energy for $\varepsilon=6 \times 10^{-4}$ generated by Fourier mode: $(1,1)$, $(1,3)$, $;(2,0), \ldots \ldots ;(0,2), \ldots \ldots$ $\ldots$; and $(0,6)$,

integrated modal energy (only the levels are different due to the summation). Consequently, we show only the evolution of the modal energy in Fig. 7 for the disturbance amplitude $\varepsilon_{2 \mathrm{D}}=\varepsilon_{3 \mathrm{D}}=6 \times 10^{-4}$, which is the case selected for the study of boundary layer noise. Note that the modal energies for the other choices of the amplitude are very similar, notably in the region $x / h \lesssim 150$, where the different Fourier components are excited. The only difference is the more or less rapid appearance of the saturation plateau, where strongly nonlinear effects are present.

As shown in Fig. 7 , the oblique mode $(1,1)$ and the $2 \mathrm{D}$ mode $(2,0)$ are initially excited. The growth of the oblique mode is greater than the plane wave, and both continue to grow for some time at a moderate rate. Later, when amplitudes of the oblique waves become considerably greater than the $2 \mathrm{D}$ wave, the local maximum of the plane wave at $x / h \simeq 45$ indicates that nonlinearity begins to appear. The streak mode $(0,2)$ then rapidly emerges and dominates the other modes. The first harmonic of the oblique waves, mode $(1,3)$, also rapidly gains several order of magnitude due to nonlinear generation. Next, higher streaks modes $(0,4)$ and $(0,6)$ are successively excited. In the late stages of transition, some oscillations around a zero growth rate are observed, corresponding to the final saturation stage.

A comparison of the evolution of Fourier components between the use of a triad and of oblique modes alone is presented in Fig. 8. The nominal case $\left(\varepsilon_{2 \mathrm{D}}=\varepsilon_{3 \mathrm{D}}=6 \times 10^{-4}\right)$ is compared to the case corresponding to Fig. 6(f), where the turbulent breakdown appears for a similar distance from the inlet. At a first glance, the $2 \mathrm{D}$ spectra are very close. Near the inlet, modes $(1,1)$ and $(1,-1)$ are dominant. The streaks mode $(0,2)$ and its harmonics appear rapidly. The only difference is that the plane wave $(2,0)$ is present from the beginning for the triad case [marked with red lozenge in Figs. 8(a)-8(c)]. We can conclude that the transition process is qualitatively unaffected and the plane wave plays the role of a catalyst that stimulates the growth of oblique waves, since a lower disturbance amplitude is necessary with the triad to obtain the same transition distance as observed for oblique waves alone. The most important mechanism of disturbance growth is connected with the transfer of energy from the primary flow to the disturbance. Energy conservation implies that the growth of one wave may be achieved as a result of energy exchange between various waves, leading to energy redistribution and the attenuation of some other waves. The present evolution of Fourier components is in qualitative agreement with the studies on oblique transition [64,77].

\section{RESULTS FOR BOUNDARY LAYER NOISE}

The large eddy simulation on the full domain, using the proposed silent inflow condition based on a triad of unstable modes for the velocity profile taken behind a backward-facing step $\left(\varepsilon_{2 \mathrm{D}}=\right.$ 


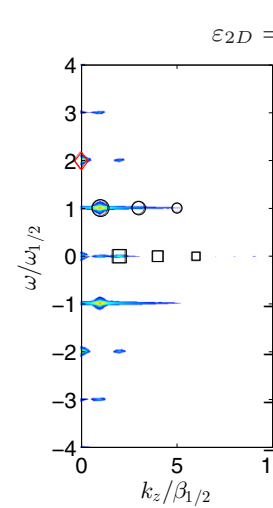

(a)
${ }_{D}=\varepsilon_{3 D}=6 \times 10^{-4}$

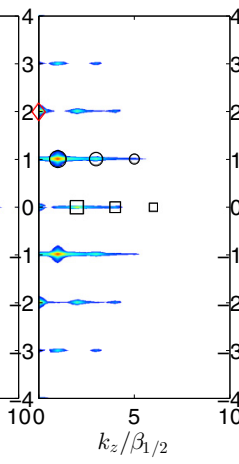

(b)

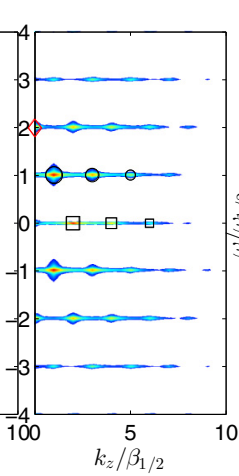

(c)

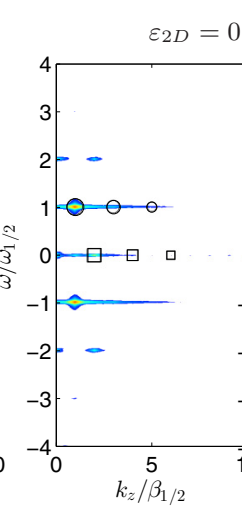

(d)

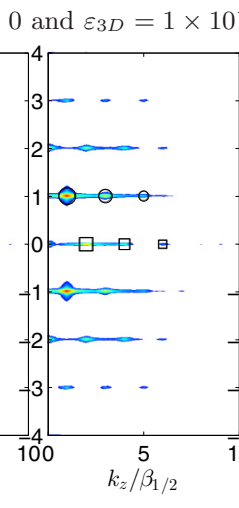

(e)

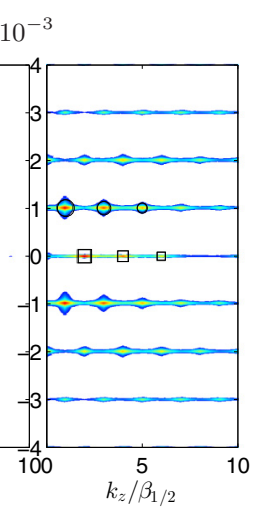

(f)

FIG. 8. Frequency wave number spectra at three successive locations: $x / h=15$ [(a), (d)], 30 [(b), (e)], and 60 [(c), (f)] for the transition from a triad of modes [(a)-(c)] and from a pair of oblique modes [(d)-(f)]. Open black circles with decreasing size are markers of oblique modes $(1,1),(1,3)$, and $(1,5)$. Open black squares with decreasing size are for streak modes $(0,2),(0,4)$, and $(0,6)$. The red lozenge marks plane mode $(2,0)$.

$\varepsilon_{3 \mathrm{D}}=6 \times 10^{-4}$ ), is now analyzed. First, the quality of the turbulent state of the boundary layer is investigated. Then, the acoustic fields are shown to demonstrate that the spurious noise is sufficiently low to study the direct emissions from the turbulent boundary layer.

\section{A. Establishment of the turbulent boundary layer}

The evolution of integral quantities is a first indicator for the establishment of a fully turbulent state. Figure 9(a) shows the evolution of the displacement and momentum thicknesses along the streamwise direction. These quantities are growing regularly beyond $x / h \approx 500$, which is the location where the turbulent flow is observed. The step height $h$, used as a reference length scale, is seen in Fig. 9(a) to correspond approximately to the initial displacement thickness. In Fig. 9(b), the Reynolds number based on the momentum thickness is plotted as function of $\operatorname{Re}_{x}$ and compared with the DNS results of FST transition by Wu and Moin [90,91] and of $K$ - and $H$-type transitions by Sayadi et al. $[92,93]$. The inlet plane for these DNS is $\operatorname{Re}_{x}=10^{5}$. For comparison purpose, the
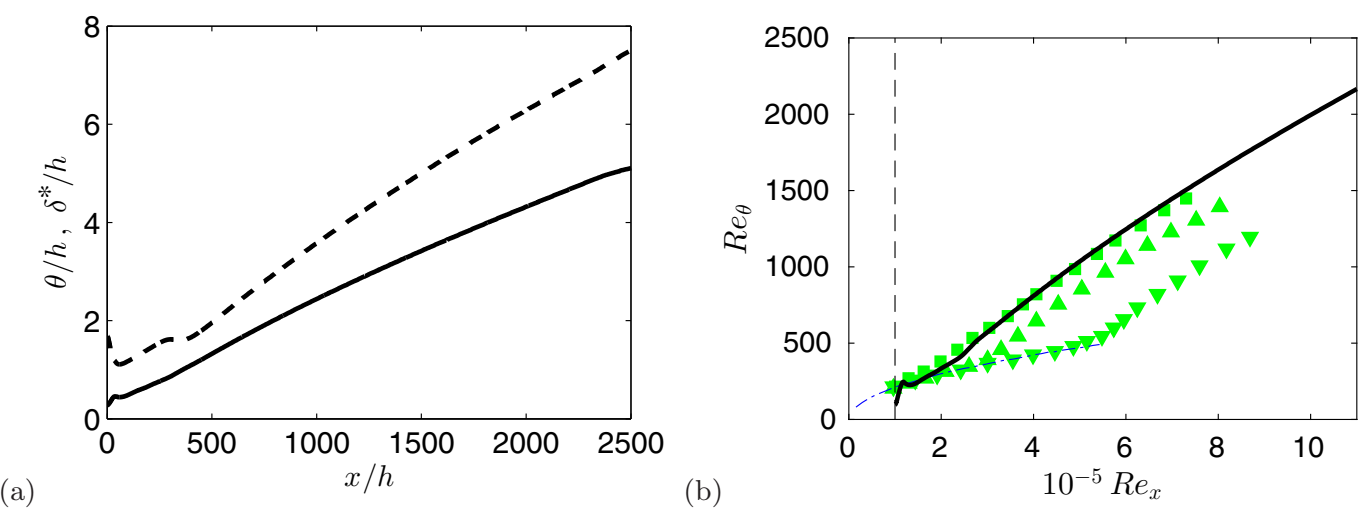

FIG. 9. (a) Displacement thickness $\left({ }_{-}{ }_{-}\right)$and momentum thickness ( ); (b) evolution of the Reynolds number based on momentum thickness $\operatorname{Re}_{\theta}$ along the plate: ), present LES; $\square$, DNS of FST transition of Wu and Moin [91]; $\triangle$, DNS of $K$ type and $\nabla, H$-type transitions of Sayadi et al. [93]; _ . - ., Blasius solution. 


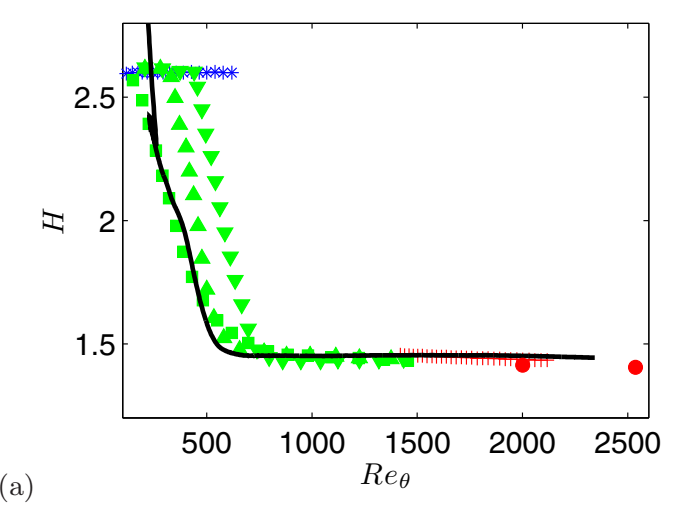

(a)

FIG. 10. (a) Shape factor $H$ as function of $\operatorname{Re}_{\theta}$ :

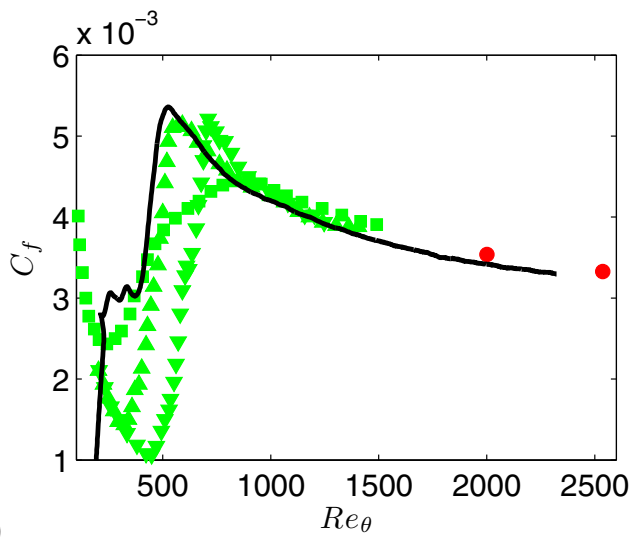

present LES; $\square$, FST [91]; $\triangle, K$-type [93]; $\nabla$,

$H$-type [93] transitions; , high-Re DNS [94]; ${ }^{*}$, Blasius solution; + , momentum integral estimate [27]. (b) Skin-friction coefficient $C_{f}$ as function of $\operatorname{Re}_{\theta}$ : — , present LES; $\square$, FST [91]; $\Delta, K$-type [93]; $\nabla, H$-type [93] transitions;, high-Re DNS [94].

inlet position of the present LES is set to $\operatorname{Re}_{x}=10^{5}$. Among the results of the Stanford group, the most rapid breakdown, marked by an almost linear growth departing from the Blasius solution, is obtained for the FST scenario followed by the $K$-type breakdown. In the $H$-type case, the value of $\operatorname{Re}_{\theta}$ remains on the laminar curve for the longest distance. The curve for the present LES is almost superimposed on the FST-transition case, indicating that the present scenario of transition occurs at faster time scale than the one for secondary instability. This can be related to the reduction of the primary linear phase due to the presence of an inflexion point in the inlet velocity profile.

The establishment of a fully turbulent boundary layer is demonstrated in Fig. 10, where the shape factor, $H$, and the skin-friction coefficient, $C_{f}$, are plotted as function of $\operatorname{Re}_{\theta}$. The shape factor in Fig. 10(a) evolves from a value higher than the laminar solution, owing to the inflectional velocity profiles downstream of the step, to an almost constant value of 1.4 , representative of zero-pressure-gradient turbulent boundary layer. The evolution also follows closely the FST DNS of $\mathrm{Wu}$ and Moin $[90,91]$. The plateau is reached for $\operatorname{Re}_{\theta} \approx 600$, faster than the $H$-type scenario. Moreover, the highest $\operatorname{Re}_{\theta}$ reached in our computation is around $\operatorname{Re}_{\theta}=2300$ and the values in this upper range are seen to slightly overestimate the DNS results of Schlatter and Örlü [94]. The skin-friction coefficient, presented in Fig. 10(b), can hardly be interpreted for $\operatorname{Re}_{\theta}<500$ since the flow is far from a canonical Blasius boundary layer. Nevertheless, the evolution of the friction coefficient clearly indicates the presence of an overshoot as in the $K$-type or $H$-type DNS of Sayadi et al. [93]. The overshoot is absent in the bypass scenario [91]. Beyond $\operatorname{Re}_{\theta} \approx 900$, all the curves show almost the same regular decrease characteristic of a fully turbulent state. Two values from the high-Re DNS of Schlatter and Örlü [94], namely $\operatorname{Re}_{\theta}=2000$ and 2540, are plotted and extend the DNS of Wu and Moin [91]. In the present computation, the values of the friction coefficient are slightly underestimated owing to the LES resolution, but the asymptotic turbulent state is satisfactorily reproduced.

The turbulent profiles are validated against published DNS results in Fig. 11. First, the mean flow provided by the present LES calculations is compared to reference data of Jimenez et al. [95] obtained at $\operatorname{Re}_{\theta}=1551$ and Schlatter and Örlü [94] at $\operatorname{Re}_{\theta}=2540$ in Fig. 11(a). In the viscous sublayer, the LES for the two selected values of $\operatorname{Re}_{\theta}$ predict fairly well the linear law. In the overlap region $\left(30<y^{+}<200\right)$, the DNS data, the log law, and the present profiles collapse. Further away from the wall, for $y^{+}>200$, the classical wake defect is visible. A slight underprediction of the friction is noticeable at the highest Reynolds number location, since our results for $\operatorname{Re}_{\theta}=2300$ 

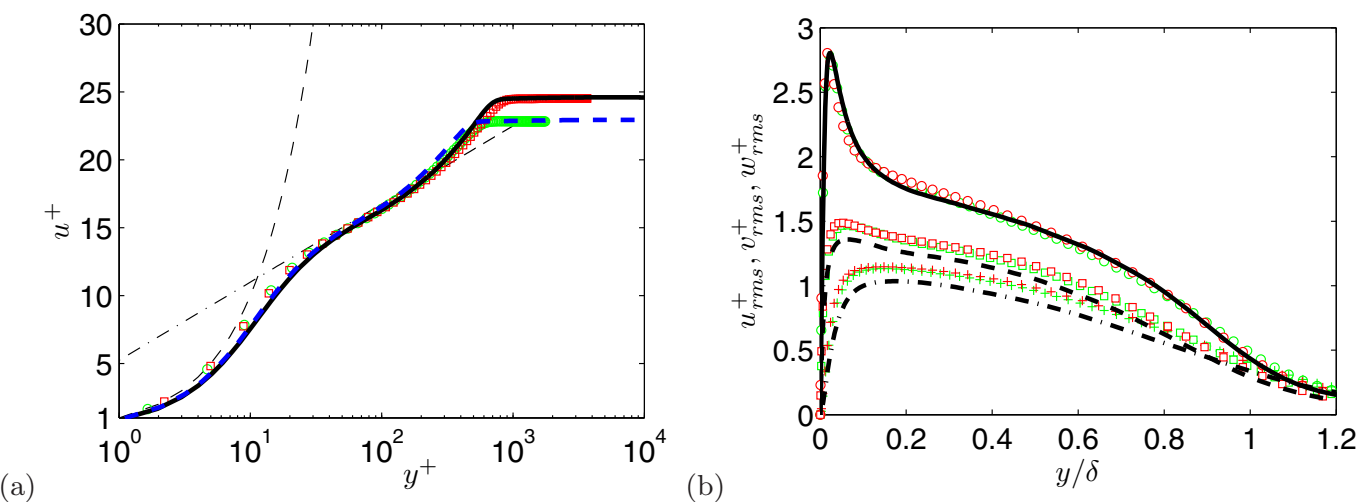

FIG. 11. (a) Profiles of the mean longitudinal velocity in wall units for $\operatorname{Re}_{\theta}=1551$ ) compared with

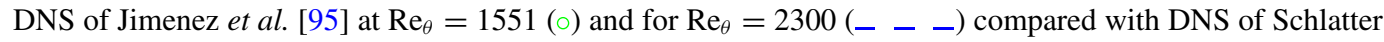
and Örlü [94] at $\operatorname{Re}_{\theta}=2540$ (०). (b) Turbulent intensities normalized by $u_{\tau}$ at $\operatorname{Re}_{\theta}=2300$ : Present LES:

$u_{\mathrm{rms}} ;-\ldots, ., v_{\mathrm{rms}} ;{ }_{-}+\ldots, w_{\mathrm{rms}}$. DNS of Jimenez at $\operatorname{Re}_{\theta}=1968: \circ, u_{\mathrm{rms}} ;+, v_{\mathrm{rms}} ; \square, w_{\mathrm{rms}}$. DNS of Schlatter at $\operatorname{Re}_{\theta}=2540$ : ०, $u_{\text {rms }} ;+, v_{\text {rms }} ; \square, w_{\text {rms }}$.

collapse with the one of Schlatter and Örlü [94] at a higher value of 2540. Figure 11(b) shows the vertical variations of the turbulent intensities. Comparisons of these distributions with those of DNS references [94,95] indicate fairly good agreement at $\operatorname{Re}_{\theta}=2300$. The tendency to underpredict the $v_{\text {rms }}$ and $w_{\text {rms }}$ is common with LES resolution [4]. The main features of a low-Reynolds-number turbulent boundary layer are satisfactorily reproduced for a range of Reynolds numbers based on the momentum thickness between 800 and 2300 .

Finally, Fig. 12 presents the successive streamwise locations, indicated by dashed lines, where one dimensional velocity spectra depicted in Fig. 13 are computed. Multiple peaks, all harmonics of $\omega_{1 / 2}$, are noticeable in the transition region. These peaks are still identifiable at $x / h=500$. In the fully turbulent boundary layer, no trace of these peaks is visible. Broadband turbulent spectra are obtained and the inertial zone, with a $-5 / 3$ slope, is limited in the present computation owing to the low Reynolds number and the cutoff of the LES grid.

\section{B. Direct noise radiation}

The fluctuating pressure field in the median plane is presented in Fig. 14. Four instants are shown to get insight into the time variation of the acoustic field. Acoustic wavefronts observed in the near field are mainly oriented in the direction opposite to the flow and have relatively large wavelengthes. These features are similar to the previous calculations in Ref. [4]. In particular, the radiation of these low-frequency sources is essentially in the upward direction due to the important Doppler

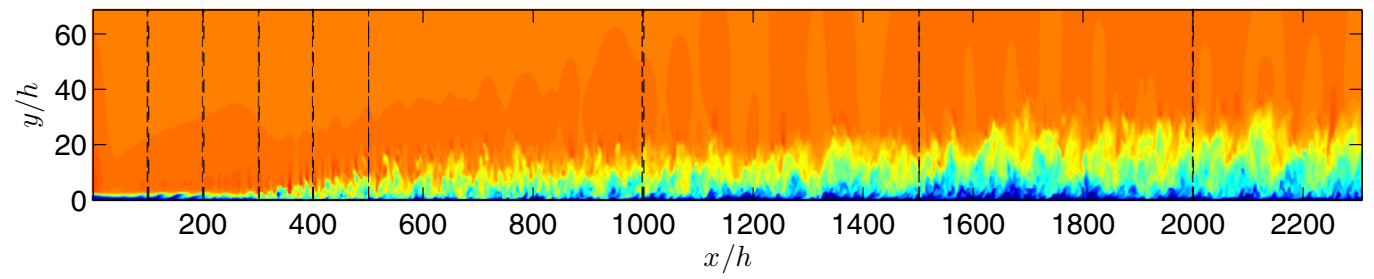

FIG. 12. Locations of the extraction planes $\left(-_{-}-\right)$for turbulent spectra: $x / h=$ $100,200,300,400,500,1000,1500,2000$, superimposed on the instantaneous streamwise velocity field. 
(a)

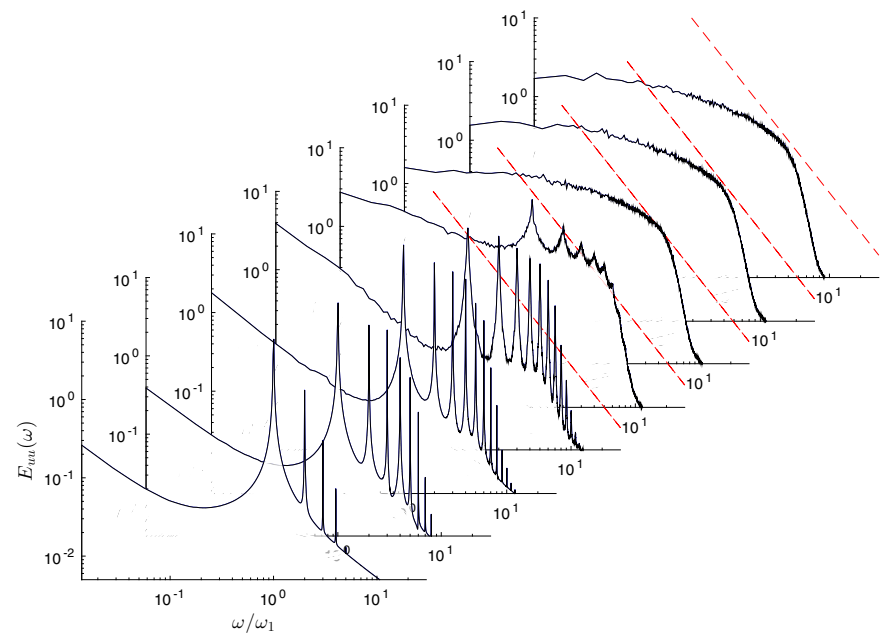

(b)
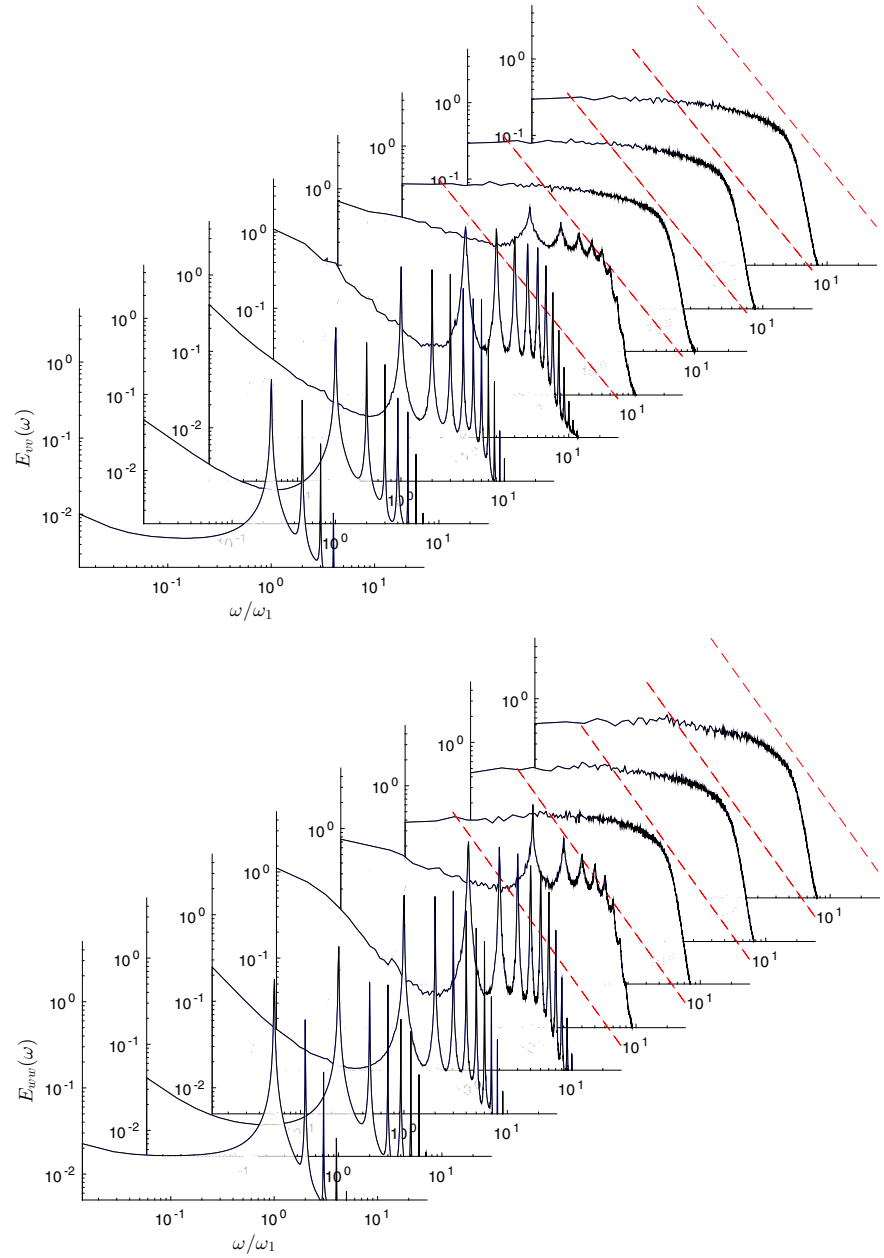

FIG. 13. One-dimensional velocity spectra for the three velocity components at $y / h \approx 0.6$ : (a) $u$; (b) $v$; (c) $w$ for the successive streamwise locations depicted in Fig. 12. The red dashed line indicates the $-5 / 3$ slope. 

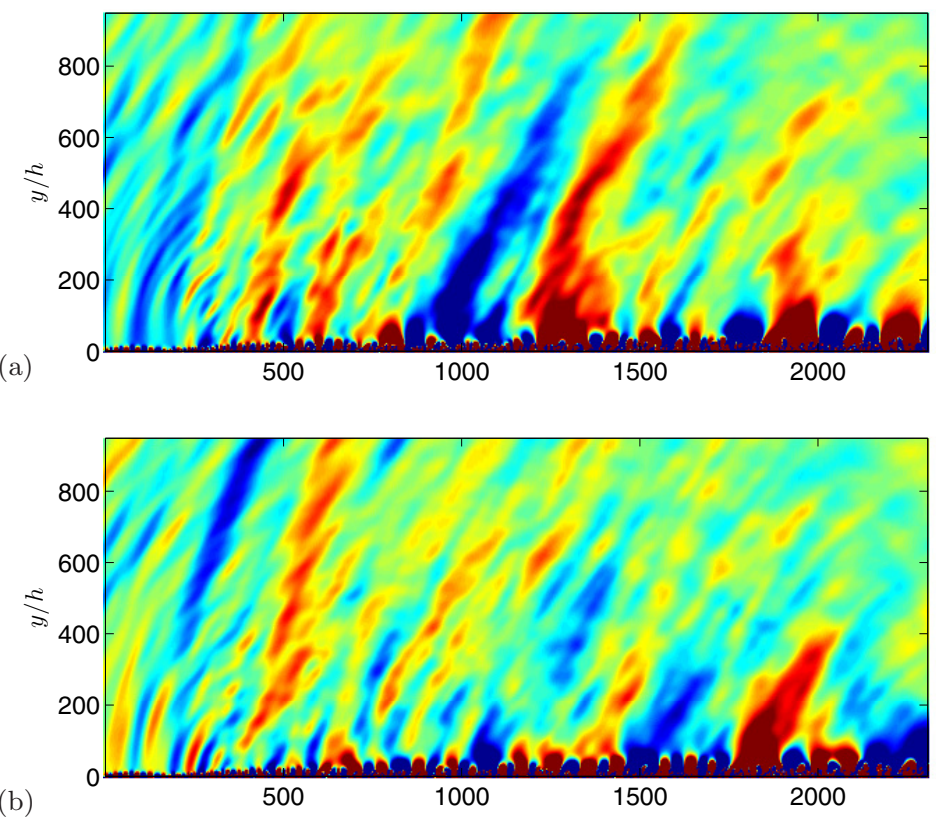

(b)
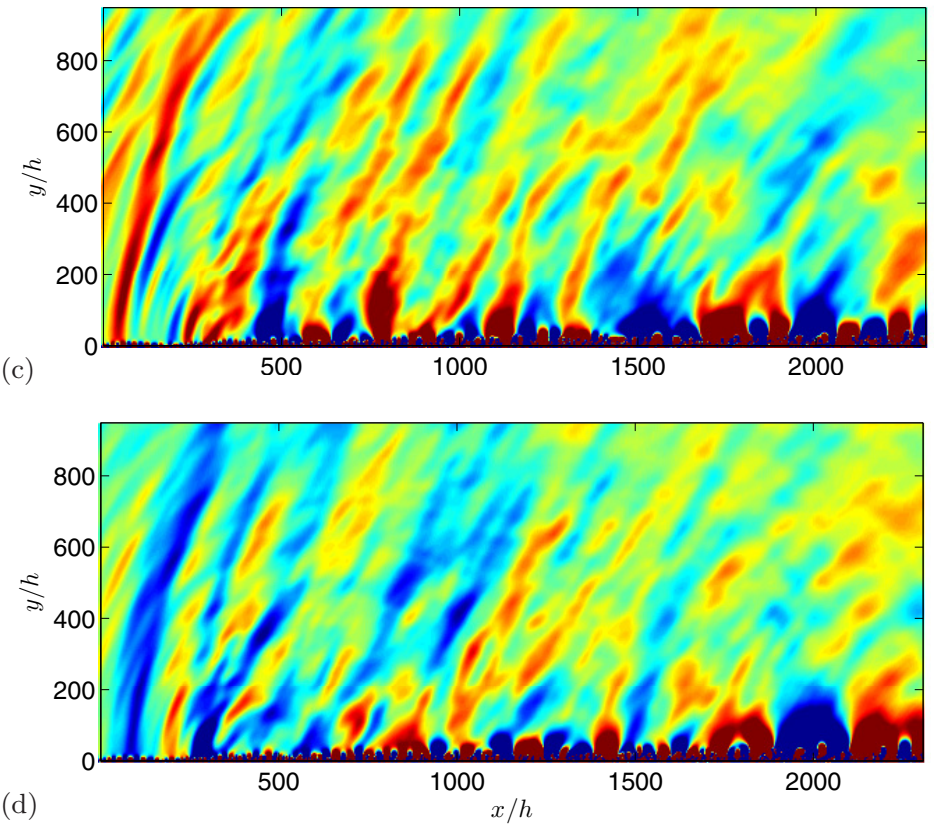

FIG. 14. Instantaneous snapshots of the pressure fluctuations $p^{\prime}$ in the median plane (levels $\pm 3 \mathrm{~Pa}$ ) at four instants.

effect at $M=0.5$, which is still reinforced for horizontal longitudinal quadrupolar sources $[4,8]$. The significant increase of upward emissions is also an indication that the sources are compact, and thus correspond to localized vortical events. Note that the new inflow condition avoids the recourse to the small step, which was seen to generate spurious high-frequency diffracted waves [4]. Here no important noise is generated by the inflow, and even if acoustics is generated by the turbulence 

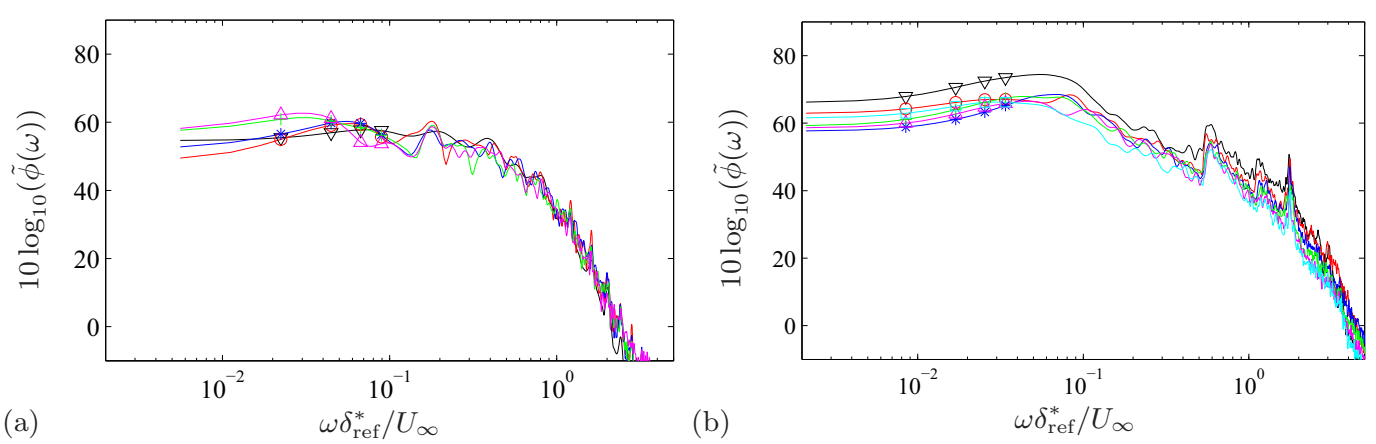

FIG. 15. Power spectral densities of the pressure perturbations $\tilde{\phi}(\omega)=\phi(\omega) U_{\infty} /\left(\rho^{2} u_{\tau}^{4} M^{2} \delta^{* 3}\right)$ in the acoustic field as a function of the Strouhal number $\omega \delta_{\text {ref }}^{*} / U_{\infty}$. (a) LES with the new inflow condition at $y / h=250$, and for various streamwise locations: $\left(\perp_{-}\right) x / h=455,\left(L_{-}\right) x / h=850,\left({ }_{-} *\right)$ $x / h=1244,\left(\__{+}\right) x / h=1638$, and $\left(\_\triangle\right) x / h=2032$. (b) Previous LES results [4] at $y / \delta_{\text {ref }}=9.6$, and for various streamwise locations: $\left(\_\nabla\right) x / \delta_{\text {ref }}=6.6,\left(\square_{-}\right) x / \delta_{\text {ref }}=19.8,\left(\square_{-} *\right) x / \delta_{\text {ref }}=33.1$, $\left(\perp_{-}+\right) x / \delta_{\text {ref }}=46.4,(\square \triangle) x / \delta_{\text {ref }}=59.7$, and $(\square \square) x / \delta_{\text {ref }}=72.9$, where subscript $\bullet_{\text {ref }}$ denotes a median boundary-layer thickness (for comparison, $y / h=250$ in subplot (a) corresponds to $y / \delta_{\text {ref }}=8.8$ ).

breakdown [see wavefronts emanating from $x / h \approx 300$ in Figs. 14(b) and 14(c)], the levels are of the same order or weaker than the direct noise from wall turbulence.

Finally, spectra of the fluctuating pressure field along the line $y / h=250$ are presented in Fig. 15(a). The spectra obtained in the previous computations [4] are reproduced in Fig. 15(b) for comparison. The broadband nature is evident in both cases. High-frequency peaks are visible in Fig. 15(b) at Strouhal numbers $\omega \delta_{\text {ref }}^{*} / U_{\infty}=0.6,1.24$, and and 1.8, which were associated to the small triggering step. They are not present in the new simulation, even if smaller peaks can be detected in the spectra at the first two locations, which are above the breakdown region $(x / h \approx 500)$. Their frequencies correspond to the one of the instability wave and its nonlinear harmonics, which break down below these stations. No trace is then visible for the acoustic spectra above the fully turbulent boundary layer, as already noted for one-dimensional velocity spectra in Fig. 13. By integrating pressure spectra, the acoustic levels at approximately ten boundary layer thicknesses from the wall represent only $0.01 \%$ of the dynamic pressure $0.5 \rho_{\infty} U_{\infty}$. This justifies the great care taken to reach a turbulent state without generating spurious noise.

\section{CONCLUSION}

A lot of studies have considered inflow turbulence generation methods, which are of practical interest for the simulation of spatially developing turbulent boundary layers or to feed a RANS/LES interface. Constraints appear when the acoustic radiation is computed in the simulation. The interaction between the inflow methods and the numerical implementation, such as the nonreflecting inlet boundary condition, needs to be taken into account to minimize spurious noise. In particular, our purpose is the study of the noise radiated directly by a turbulent boundary layer on a flat plate, which has a very low efficiency. The design of a silent inflow technique to introduce perturbations necessary to trigger laminar-to-turbulent transition becomes a critical point.

An inflow strategy based on transition by modal interaction has been developed. A preliminary steady two-dimensional simulation is performed for the flow over a backward-facing step with a height representative of tripping band used in experiments. A base flow is defined by extracting flow variables in a vertical plane located few points downstream of the step and a compressible linear stability analysis is conducted to find a resonant triad of unstable modes. The triad is made up of one two-dimensional wave and two oblique subharmonic waves with opposite phase angles satisfying the resonant condition of Craik [78]. In our application, the velocity profile exhibits an inflexion point, 
yielding unstable modes with a greater growth rate, which is favorable for developing instability waves from a low initial amplitude. These disturbance waves are entered in the inlet boundary condition of Tam and Dong to trigger the transition to turbulence. An important point to reduce spurious noise is to prescribe correctly the thermodynamic variables associated with the velocity disturbances. In our approach, the thermodynamic disturbances are known since a compressible stability solver is used.

Two principal points have been checked: (i) that the transition is smooth and quiet acoustically and (ii) that a fully turbulent state is reached. For that, a compressible large eddy simulation was carried out at Mach number 0.5. With the present tripping method, the transition is dominated by the oblique modes which rapidly induce the onset of streaks that further break down into turbulence by a liftup mechanism. The presence of the fundamental plane wave in the triad favors the resonant interaction, producing rapid growth of wave amplitudes. This $O$-type scenario pertains to the class of subharmonic transition. Notably staggered $\Lambda$-shape vortices are rapidly formed before the onset of low-speed streaks. The transition is characterized by a continuous spectral enrichment and it has been checked that a fully turbulent state with no trace of the initial disturbances is reached for $\operatorname{Re}_{\theta} \approx 900$. At the end of the computational domain, a Reynolds number of 2300 is reached. Turbulent statistics are in fairly good agreement with DNS references. Finally, the direct acoustic field is well retrieved by the present approach and is not perturbed by spurious noise due to the inflow technique.

This silent inflow has been used successfully to investigate the properties of wall pressure in the acoustic domain, that is the range of supersonic wave numbers in a frequency-wave-number representation of the wall pressure [96,97]. The trace of acoustic wave numbers in these direct noise computations is consistent with the levels of direct radiation outside the turbulent boundary layer. Furthermore, the present technique can also give access to the decay rate in the subconvective wave numbers, which is hardly reproduced in available models or in experiments. In future studies, the silent inflow method will be used to investigate the noise generated in the transition process, which can give further insights into the coherent structures responsible for noise emissions. In a more general manner, the proposed inflow condition can be used or adapted to study the noise generation in configurations where a spatially developing turbulent flow is incoming, such as cavity flows or inlet conditions in jet nozzles.

\section{ACKNOWLEDGMENTS}

This research has been funded by the Agence Nationale de la Recherche (ANR) through the ANR-2011-BS09-035-01 project SONOBL. This work was granted access to the HPC resources of IDRIS and CCRT under the allocation 2016-1736 made by GENCI (Grand Equipement National de Calcul Intensif).

\section{APPENDIX A: LOCAL LINEAR STABILITY ANALYSIS}

\section{Equations}

The stability equations are derived starting from the three-dimensional linearized Navier-Stokes equations in Cartesian coordinates $(x, y, z)$. In the present study, the base flow is provided by results of the 2D flow solver presented in the previous section and interpolated on the stability grid. The local assumption imposes that base flow quantities are function of $y$ solely and the solution can be written in normal mode form:

$$
\mathbf{q}^{\prime}(x, y, z, t)=\frac{1}{(2 \pi)^{3}} \int_{F_{\omega}}\left[\int_{L_{\beta}}\left(\int_{L_{\alpha}} \tilde{\mathbf{q}}(y ; \alpha, \beta, \omega) e^{i(\alpha x+\beta z-\omega t)} d \alpha\right) d \beta\right] d \omega+\text { c.c. }
$$


with $\mathbf{k}=\alpha \mathbf{e}_{x}+\beta \mathbf{e}_{z}$ being the a priori complex wave number vector and $\omega$ being the circular frequency. $F_{\omega}, L_{\beta}$ and $L_{\alpha}$ are complex integration contours. These contours are chosen to verify the causality and define the inverse Fourier transform.

The normal mode form is then introduced in the linearized Navier-Stokes equations to obtain the stability equations. The boundary conditions at the freestream $(y \rightarrow \infty)$ and at the wall $(y=0)$ are as follows:

$$
\tilde{u}=\tilde{v}=\tilde{w}=0, \frac{\partial \tilde{p}}{\partial y}=0, \text { and } \frac{\partial \tilde{T}}{\partial y}=0 \text { as } y \rightarrow 0, \infty .
$$

As both the equations and the boundary conditions are homogeneous, the system constitutes an eigenvalue problem. The parameters of the problem are $\omega, \beta, M$, and Re (eventually $\operatorname{Pr}$ ), whereas $\alpha=\alpha_{r}+i \alpha_{i}$ is the eigenvalue. Formally the system of stability equations can be written as

$$
\mathbf{A}_{2} \frac{d^{2} \tilde{\mathbf{q}}}{d y^{2}}+\mathbf{A}_{1} \frac{d \tilde{\mathbf{q}}}{d y}+\mathbf{A}_{0} \tilde{\mathbf{q}}=0
$$

where $\tilde{\mathbf{q}}=(\tilde{u}, \tilde{v}, \tilde{w}, \tilde{p}, \tilde{T})^{T}$ and $\mathbf{A} \bullet \in \mathcal{M}_{5}(\mathbb{C})$. The system (A3) is a differential eigenvalue problem.

\section{Numerical method}

The eigenvalue problem (A3) is solved using the Chebyshev collocation spectral method. The physical domain $0 \leqslant y / h \leqslant+y_{\max }$ is now transformed into the computational domain. We use $N$ th-order Chebyshev polynomials $T_{N}$ defined in the interval $-1 \leqslant \zeta_{j} \leqslant+1$ where the collocation points $\zeta_{j}$ are the extrema of $T_{n}$ and are given by $\zeta_{j}=\cos (\pi j / N)$ where $j=0, \ldots, N$. In order to apply the spectral collocation method, an interpolant polynomial is constructed for the dependent variables in terms of their values at the collocation points. Thus an $N$ th-order polynomial may be written as

$$
\tilde{\mathbf{q}}(\zeta)=\sum_{j=0}^{N} K_{j}(\zeta) \tilde{\mathbf{q}}_{j}\left(\zeta_{j}\right)
$$

where the interpolant $K_{j}(\zeta)$ for the Chebyshev scheme is given by

$$
K_{j}(\zeta)=\left(\frac{1-\zeta_{j}^{2}}{\zeta-\zeta_{j}}\right) \frac{T_{N}^{\prime}(\zeta)}{N^{2} c_{j}}(-1)^{k+1}, \text { where } c_{0}=c_{N}=2, c_{k}=1, \text { for } k \in\{1, \ldots, N-1\}
$$

The first derivative of $\tilde{\mathbf{q}}(\zeta)$ may be written as

$$
\frac{d \tilde{\mathbf{q}}}{d \zeta}\left(\zeta_{j}\right)=\sum_{k=0}^{N} E_{j k} \tilde{\mathbf{q}}\left(\zeta_{k}\right)
$$

where $E_{j k}$ are the elements of the derivative matrix given by

$$
E_{j k}=\frac{c_{j}}{c_{k}} \frac{(-1)^{j+k}}{\zeta_{j}-\zeta_{k}}, j \neq k, E_{j j}=-\frac{\zeta_{j}}{2\left(1-\zeta_{j}^{2}\right)}, E_{00}=\frac{2 N^{2}+1}{6}=-E_{N N} .
$$

The scaling factor for the transformation between physical and computational domains is given as $S_{j}=(d \zeta / d y)_{j}, j=0, \ldots, N$; then the first derivative matrix $\mathbf{F}$ in the physical domain may be written as

$$
F_{j k}=S_{j} E_{j k}
$$


and the second derivative matrix $G_{j k}$ is simply $G_{j k}=F_{j m} F_{m k}$. Now the stability equation (A3) may be written at the collocation points $\zeta_{j}$ as

$$
\left(A_{2}\right)_{j} \sum_{k=0}^{N} G_{j k} \tilde{\mathbf{q}}_{k}+\left(A_{1}\right)_{j} \sum_{k=0}^{N} F_{j k} \tilde{\mathbf{q}}_{k}+\left(A_{0}\right)_{j} \tilde{\mathbf{q}}_{j}=0 .
$$

The Chebyshev interval $-1 \leqslant \zeta_{j} \leqslant+1$ is transformed to the computational domain $0 \leqslant y / h \leqslant$ $+y_{\max }$ by use of the mapping

$$
y_{j}=y\left(\zeta_{j}\right)=\frac{a_{0}\left(1-\zeta_{j}\right)}{a_{1}+\zeta_{j}} \quad \text { with } \quad a_{0}=\frac{y_{a} y_{\max }}{\left(y_{\max }-2 y_{a}\right)}, a_{1}=1+\frac{2 a_{0}}{y_{\max }},
$$

where $y_{a}$ corresponds to the height from the wall where $50 \%$ of the points are located. Here, $y_{\max }=$ $60, N=100$, and $y_{a}=6$.

\section{Eigenvalue problem}

The discretized eigenproblem (A4) can be written in the following form:

$$
\left[\mathbf{C}_{0}+\mathbf{C}_{1} \alpha+\mathbf{C}_{2} \alpha^{2}\right] \tilde{\phi}(y)=0, \tilde{\phi}=\left(\tilde{\mathbf{q}}_{0}, \ldots, \tilde{\mathbf{q}}_{N}\right)^{t},
$$

where (A5) is a nonlinear problem for the eigenvalue $\alpha$. In order to transform this problem into a problem with linear eigenvalues, the following variable transformation is used, $\mathcal{Q}=\left(\tilde{\phi}, \tilde{\phi}_{1}=\alpha \tilde{\phi}\right)^{t}$, and the system (A5) becomes

$$
(\mathbf{A}-\alpha \mathbf{B}) \mathcal{Q}=0
$$

with

$$
\mathbf{A}=\left(\begin{array}{cc}
0 & 1 \\
\mathbf{C}_{0} & \mathbf{C}_{1}
\end{array}\right), \mathbf{B}=\left(\begin{array}{cc}
1 & 0 \\
0 & -\mathbf{C}_{2}
\end{array}\right)
$$

Since the matrix $\mathbf{B}$ is not invertible, an attractive and popular technique is to apply first the shift to the original problem and then carry out the shift invert. Specifically, let $\sigma$ be a user-selected shift such that the matrix $\mathbf{A}-\sigma \mathbf{B}$ is nonsingular; then the original problem (A6) can be transformed to

$$
(\mathcal{A}-\xi \mathbf{I}) \mathcal{Q}=\mathbf{0}
$$

where $\xi=1 /(\alpha-\sigma)$ and $\mathcal{A}=(\mathbf{A}-\sigma \mathbf{B})^{-1} \mathbf{B}$. A QZ algorithm type is then used to solve the eigenvalue problem (A8). Newton's algorithm is implemented to follow the branches.
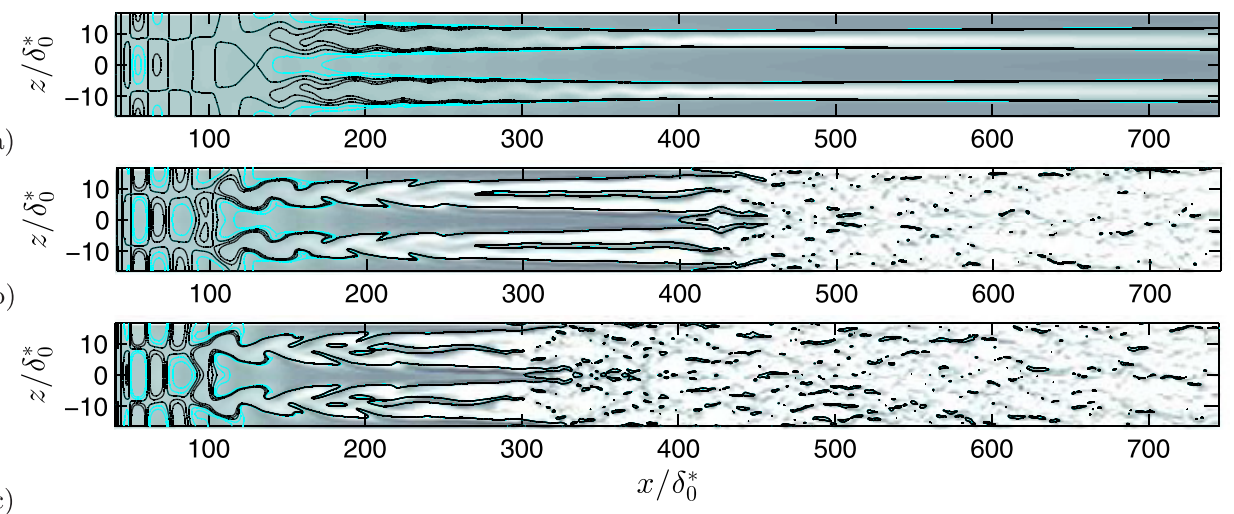

FIG. 16. Instantaneous plots of the streamwise velocity in a streamwise-spanwise plane at $y=0.77 \delta_{0}^{*}$ for increasing values of the forcing amplitude: $\varepsilon_{3 \mathrm{D}}=8 \times 10^{-3}(\mathrm{a}), 2 \times 10^{-2}(\mathrm{~b})$, and $3 \times 10^{-2}$ (c). 

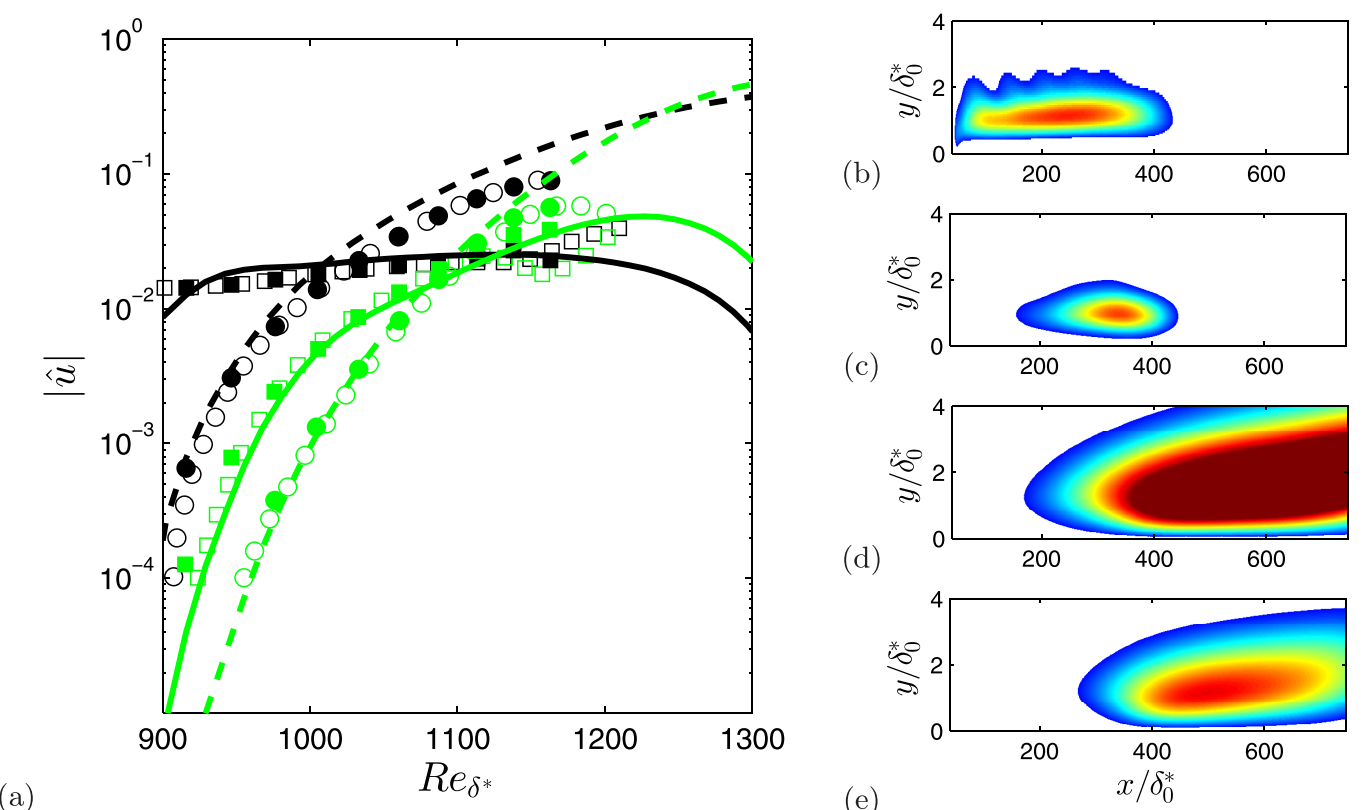

(b)

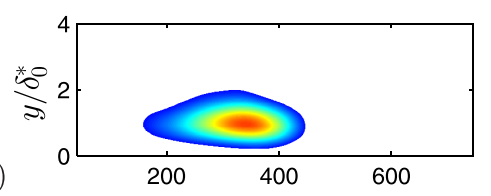

(c)

(d)

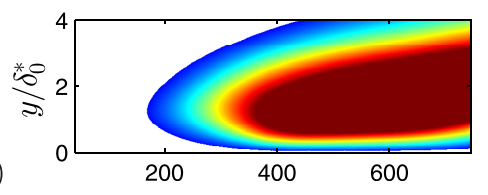

(e)

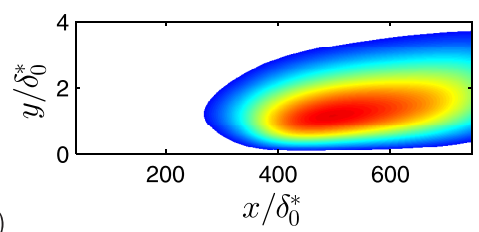

FIG. 17. Amplitude growth of streamwise velocity modes for an oblique-wave pair with amplitude $\varepsilon=$ $8 \times 10^{-3}$ (a). Lines represent the present computation, open symbols represent the DNS of Joslin et al., and filled symbols represent the PSE of Joslin et al.: mode $(1,1)\left(\_, \square, \mathbf{\square}\right)$, mode $(1,3)(\square, \square, \square)$, mode $(0,2)$ $\left({ }_{-}{ }_{-}, \circ, \bullet\right)$, and mode $(0,4)\left({ }_{-}{ }_{-}, \circ, \bullet\right)$. Maps of the modes $(1,1)(b),(1,3)(c),(0,2)(d)$, and $(0,4)(e)$. Color levels of $|\hat{u}|$ are between 0 and 0.03 for panels (b) and (c) and between 0 and 0.3 for panels (d) and (e).

\section{APPENDIX B: VALIDATION OF OBLIQUE-WAVE BREAKDOWN}

One of the few existing study of oblique-wave breakdown in a spatially developing boundary layer has been carried out by Joslin et al. [81]. The transition from a pair of oblique waves in Ref. [81] was computed by both spatial DNS and PSE. The profiles of the oblique-wave pair was obtained from linear-stability analysis for an incompressible Blasius boundary-layer flow at a Reynolds number $\mathrm{Re}_{\delta_{0}^{*}}=900$, based on the initial displacement thickness. The results are nondimensionalized with respect to the freestream velocity $U_{\infty}$ and the displacement thickness $\delta_{0}^{*}$. The selected characteristics of the oblique waves were frequency $\omega=0.0774$ and spanwise wave numbers $\beta= \pm 0.2$. In order to validate the present inflow condition and the resolution capability of our compressible solver, the case of Joslin et al. [81] is reproduced. First, a laminar boundary-layer base flow is computed at a Mach number $M=0.1$, since a compressible solver is used. Comparisons with Blasius's solution show that we can assume that the base flow is a Blasius boundary layer. The Reynolds number based on the initial displacement thickness is close to that used by Joslin et al., $\operatorname{Re}_{\delta_{0}^{*}} \simeq 903$. The linear-stability solver described in Appendix A is used to determine oblique modes with $\omega=0.0774$. The complex streamwise wave number has a real part $\alpha_{r} \simeq 0.20366$ and an imaginary part $\alpha_{i} \simeq-0.00227$. The wave angle is $44.6^{\circ}$, yielding a spanwise wave number $\beta= \pm 0.2008$, close to the value used by Joslin et al..

The input modes $(1,1)$ and $(1,-1)$, corresponding to $(\omega, \beta)$ and $(\omega,-\beta)$, are introduced in the inflow boundary condition, as described by Eq. (2). The computations are performed on a grid of 700 uniformly spaced streamwise points, 150 wall-normal points with a grid stretching from the wall similar to that described in Sec. IV, and 100 uniformly spaced spanwise points. The length of the domain is 745 and its height is 35 , expressed with respect to the initial displacement thickness. The spanwise extent is taken equal to one spanwise wavelength of the input modes, $\lambda_{z}=2 \pi / \beta$. 
Results for the streamwise velocity fluctuations are presented in Fig. 16 for three increasing values of the forcing amplitude $\varepsilon_{3 \mathrm{D}}$. Just after the inlet, the checkerboard pattern due to the interaction of the two initial oblique waves with opposite angles is seen to decay slowly. Further downstream, the pattern changes with higher spanwise wave numbers and the nonlinear interaction of the oblique waves generates counter-rotating streamwise vortices. This first development phase is similar to the observations of Berlin et al. [64]. The low-speed streaks have then a sinuous evolution with an eventual wavelength doubling and the appearance of high frequencies, leading rapidly to turbulence. At the lowest level, $\varepsilon_{3 \mathrm{D}}=8 \times 10^{-3}$ in Fig. 16(a), the breakdown to turbulence is not observed in the computational box. This case is used for comparison with the results of Joslin et al. [81] for their larger disturbance amplitude, namely 0.01 for the normalized root-mean-square value of streamwise velocity fluctuations of oblique modes. The evolutions of Fourier components obtained for the present computation are compared in Fig. 17 with the DNS and PSE results of Ref. [81]. The quantitative agreement is fairly good. The initial oblique modes $(1,1)$ grow slowly, whereas the streak mode $(0,2)$ rapidly emerges because of the self-interaction of the oblique waves. The interaction of the oblique modes with these streamwise vortices generates amplified harmonic wave $(1,3)$, and the self-interaction of the streak mode supplies energy to the $(0,4)$ mode. Some discrepancies are visible notably near the downstream end of data of Joslin et al., since their DNS computations are under-resolved in this region and the limits of PSE theory are maybe reached. Moreover, the numerical compressible inflow condition is not perfect and the waves are entered after a phase of adaptation. The qualitative and quantitative similarities of modal interactions are nevertheless sufficient to give confidence in the present numerical solver to reproduce an oblique-wave transition.

[1] X. Gloerfelt and T. Le Garrec, Generation of inflow turbulence for aeroacoustic applications, in AIAA/CEAS Aeroacoustics Conference (29th AIAA Aeroacoustics Conference), 5-7 May, 2008, Vancouver, Canada (AIAA, Reston, VA, 2008), paper AIAA 2008-2926.

[2] G. Aubard, X. Gloerfelt, and J.-C. Robinet, Characterisation of synthetic turbulence methods for large-eddy simulation of supersonic boundary layers, in Progress in Turbulence and Wind Energy IV, edited by M. Oberlack, J. Peinke, A. Talamelli, L. Castillo, and M. Hölling, Springer Proceedings in Physics, Vol. 141 (Springer, Berlin, Heidelberg, 2012), pp. 81-84.

[3] J. Chicheportiche and X. Gloerfelt, Effect of a turbulent incoming boundary layer on noise radiation by the flow over cylindrical cavities, in 17th AIAA/CEAS Aeroacoustics Conference (32nd AIAA Aeroacoustics Conference), 5-8 June, 2011, Portland, Oregon (AIAA, Reston, VA, 2011), paper AIAA 2011-2862.

[4] X. Gloerfelt and J. Berland, Turbulent boundary-layer noise: Direct radiation at Mach number 0.5, J. Fluid Mech. 723, 318 (2013).

[5] A. Powell, Aerodynamic noise and the plane boundary, J. Acoust. Soc. Am. 32, 982 (1960).

[6] N. Curle, The influence of solid boundaries upon aerodynamic sound, Proc. R. Soc. London, Ser. A 231, 505 (1955).

[7] X. Gloerfelt and J. Berland, Direct computation of turbulent boundary layer noise, in 15th AIAA/CEAS Aeroacoustics Conference (30th AIAA Aeroacoustics Conference), 11-13 May, 2009, Miami, Florida (AIAA, Reston, VA, 2009), paper AIAA 2009-3401.

[8] X. Gloerfelt, The link between wall pressure spectra and radiated sound from turbulent boundary layers, in 16th AIAA/CEAS Aeroacoustics Conference, 7-9 June, 2010, Stockholm, Sweden (AIAA, Reston, VA, 2010), paper AIAA 2010-3904.

[9] M. S. Howe, Surface pressures and sound produced by turbulent flow over smooth and rough walls, J. Acoust. Soc. Am. 90, 1041 (1991).

[10] G. Aubard, X. Gloerfelt, and J.-C. Robinet, Large-eddy simulation of broadband unsteadiness in a shock/boundary-layer interaction, AIAA J. 51, 2395 (2013). 
[11] X. Gloerfelt and T. Le Garrec, Trailing edge noise from an isolated airfoil at a high Reynolds number, in 15th AIAA/CEAS Aeroacoustics Conference (30th AIAA Aeroacoustics Conference), 11-13 May, 2009, Miami, Florida (AIAA, Reston, VA, 2009), paper AIAA 2009-3201.

[12] T. Colonius and S. K. Lele, Computational aeroacoustics: Progress on nonlinear problems of sound generation, Progr. Aerospace Sci. 40, 345 (2004).

[13] M. Wang, J. B. Freund, and S. K. Lele, Computational prediction of flow-generated sound, Annu. Rev. Fluid Mech. 38, 483 (2006).

[14] S. K. Lele and J. W. Nichols, A second golden age of aeroacoustics?, Philos. Trans. R. Soc. London, Ser. A 372, 20130321 (2014).

[15] C. Bogey and C. Bailly, A family of low dispersive and low dissipative explicit schemes for noise computation, J. Comput. Phys. 194, 194 (2004).

[16] K. W. Thompson, Time-dependent boundary conditions for hyperbolic systems, J. Comput. Phys. 68, 1 (1987).

[17] T. J. Poinsot and S. K. Lele, Boundary conditions for direct simulations of compressible viscous flows, J. Comput. Phys. 101, 104 (1992).

[18] C. K. W. Tam and Z. Dong, Radiation and outflow boundary conditions for direct computation of acoustic and flow disturbances in a nonuniform mean flow, J. Comput. Acous. 4, 175 (1996).

[19] C. K. W. Tam, Advances in numerical boundary conditions for computational aeroacoustics, J. Comput. Acous. 6, 377 (1998).

[20] A. Keating, U. Piomelli, E. Balaras, and H.-J. Kaltenbach, A priori and a posteriori tests of inflow conditions for large-eddy simulations, Phys. Fluids 16, 4696 (2004).

[21] G. R. Tabor and M. H. Baba-Ahmadi, Inlet conditions for large eddy simulation: A review, Comp. Fluids 39, 553 (2010).

[22] X. Wu, Inflow turbulence generation methods, Annu. Rev. Fluid Mech. 49, 23 (2017).

[23] N. Hutchins, Caution: Tripping hazards, J. Fluid Mech. 710, 1 (2012).

[24] P. Schlatter and R. Örlü, Turbulent boundary layers at moderate Reynolds numbers: Inflow length and tripping effects, J. Fluid Mech. 710, 5 (2012).

[25] X. Gloerfelt, C. Bogey, and C. Bailly, Numerical evidence of mode switching in the flow-induced oscillations by a cavity, Int. J. Aeroacoustics 2, 193 (2003).

[26] S. Lee, S. K. Lele, and P. Moin, Simulation of spatially evolving turbulence and the applicability of Taylor hypothesis in compressible flow, Phys. Fluids A 4, 1521 (1992).

[27] T. S. Lund, X. Wu, and K. D. Squires, Generation of turbulent inflow data for spatially developing boundary layer simulations, J. Comput. Phys. 140, 233 (1998).

[28] H. Le, P. Moin, and J. Kim, Direct numerical simulation of turbulent flow over a backward-facing step, J. Fluid Mech. 330, 349 (1997).

[29] Y. Na and P. Moin, Direct numerical simulation of a separated turbulent boundary layer, J. Fluid Mech. 374, 379 (1998).

[30] Y. M. Chung and H. J. Sung, Comparative study of inflow conditions for spatially evolving simulation, AIAA J. 35, 269 (1997).

[31] R. Kraichnan, Diffusion by a random velocity field, Phys. Fluids 13, 22 (1970).

[32] W. Béchara, C. Bailly, P. Lafon, and S. Candel, Stochastic approach to noise modeling for free turbulent flows, AIAA J. 32, 455 (1994).

[33] A. Smirnov, S. Shi, and I. Celik, Random flow generation technique for large eddy simulation and particle-dynamics modeling, Trans. ASME: J. Fluids Eng. 123, 359 (2001).

[34] M. Billson, L.-E. Eriksson, and L. Davidson, Modeling of synthetic anisotropic turbulence and its sound emission, in 10th AIAA/CEAS Aeroacoustics Conference, Aeroacoustics Conferences (AIAA, Reston, VA, 2004), paper AIAA 2004-2857.

[35] N. D. Sandham, Y. F. Yao, and A. A. Lawal, Large-eddy simulation of transonic turbulent flow over a bump, Int. J. Heat Fluid Flow 24, 584 (2003).

[36] R. D. Sandberg and N. D. Sandham, Direct numerical simulation of turbulent flow past a trailing edge and the associated noise generation, J. Fluid Mech. 596, 353 (2008). 
[37] O. Marsden, C. Bogey, and C. Bailly, Investigation of flow features around shallow round cavities subject to subsonic grazing flow, Phys. Fluids 24, 125107 (2012).

[38] N. Jarrin, S. Benhamadouche, D. Laurence, and R. Prosser, A synthetic-eddy-method for generating inflow conditions for large-eddy simulation, Int. J. Heat Fluid Flow 27, 585 (2006).

[39] M. Pamiès, P. E. Weiss, E. Garnier, S. Deck, and P. Sagaut, Generation of synthetic turbulent inflow data for large eddy simulation of spatially evolving wall-bounded flows, Phys. Fluids 21, 045103 (2009).

[40] M. Klein, A. Sadiki, and J. Janicka, A digital filter based generation of inflow data for spatially developing direct numerical or large eddy simulations, J. Comput. Phys. 186, 652 (2003).

[41] L. di Mare, M. Klein, P. Jones, and J. Janicka, Synthetic turbulence inflow conditions for large-eddy simulation, Phys. Fluids 18, 025107 (2006).

[42] I. Veloudis, Z. Yang, J. J. McGuirk, G. J. Page, and A. Spencer, Novel implementation and assessment of a digital filter based approach for the generation of LES inlet conditions, Flow, Turbulence Combust. 79, 1 (2007).

[43] E. Touber and N. D. Sandham, Large-eddy simulation of low-frequency unsteadiness in a turbulent shock-induced separation bubble, Theor. Comput. Fluid Dyn. 23, 79 (2009).

[44] P. Subbareddy, D. Peterson, G. V. Candler, and I. Marusic, A synthetic inflow generation method using the attached eddy hypothesis, in 24th AIAA Applied Aerodynamics Conference, 5-8 June, 2006, San Francisco, California (AIAA, Reston, VA, 2006), paper AIAA 2006-3672.

[45] P. R. Spalart, Direct simulation of a turbulent boundary layer up to $\operatorname{Re}_{\theta}=1410$, J. Fluid Mech. 187, 61 (1988).

[46] P. Flohr and E. Balaras, Large-eddy simulation of a turbulent boundary layer, Technical Note 187, von Kármán Institute for Fluid Dynamics, Rhode Saint Genese Belgium, 1995 (unpublished).

[47] K. Liu and R. H. Pletcher, Inflow conditions for the large eddy simulation of turbulent boundary layers: A dynamic recycling procedure, J. Comput. Phys. 219, 1 (2006).

[48] A. Ferrante and S. E. Elghobashi, A robust method for generating inflow conditions for direct simulations of spatially developing turbulent boundary layers, J. Comput. Phys. 198, 372 (2004).

[49] G. Araya, L. Castillo, C. Meneveau, and K. Jansen, A dynamic multi-scale approach for turbulent inflow boundary conditions in spatially developing flows, J. Fluid Mech. 670, 581 (2011).

[50] S. Stolz and N. A. Adams, Large-eddy simulation of high-Reynolds-number supersonic boundary layers using the approximate deconvolution model and rescaling and recycling technique, Phys. Fluids 15, 2398 (2003).

[51] S. Xu and M. Martin, Assessment of inflow boundary conditions for compressible turbulent boundary layers, Phys. Fluids 16, 2623 (2004).

[52] P. Sagaut, E. Garnier, E. Tromeur, L. Larchevêque, and E. Labourasse, Turbulent inflow conditions for large-eddy simulation of compressible wall-bounded flows, AIAA J. 42, 469 (2004).

[53] A. Spille-Kohoff and H.-J. Kaltenbach, Generation of turbulent inflow data with a prescribed shear-stress profile, in DNS/LES Progress and Challenges, Proceedings of the Third AFOSR International Conference on Direct Numerical Simulation and Large Eddy Simulation (TAICDL) (Greyden Press, Dayton, Ohio, 2001), pp. 1-4, http://www.dtic.mil/dtic/tr/fulltext/u2/p013648.pdf.

[54] R. Laraufie, S. Deck, and P. Sagaut, A dynamic forcing method for unsteady turbulent inflow conditions, J. Comput. Phys. 230, 8647 (2011).

[55] P. Druault, S. Lardeau, J.-P. Bonnet, F. Coiffet, J. Delville, E. Lamballais, J. Largeau, and L. Perret, Generation of three-dimensional turbulent inlet conditions for large-eddy simulation, AIAA J. 42, 447 (2004).

[56] L. Perret, J. Delville, R. Manceau, and J.-P. Bonnet, Generation of turbulent inflow conditions for large-eddy simulation from stereoscopic PIV measurements, Int. J. Heat Fluid Flow 27, 576 (2006).

[57] Z. Xiong, S. Nagarajan, and S. Lele, Simple method for generating inflow turbulence, AIAA J. 42, 2164 (2004).

[58] P. Johansson and H. Andersson, Generation of inflow data for inhomogeneous turbulence, Theor. Comput. Fluid Dyn. 18, 371 (2004).

[59] P. J. Schmid and D. S. Henningson, Stability and Transition in Shear Flows, Applied Mathematical Sciences Vol. 142 (Springer, Berlin, 2001). 
[60] P. S. Klebanoff, K. D. Tidstrom, and L. M. Sargent, The three-dimensional nature of boundary layer instability, J. Fluid Mech. 12, 1 (1962).

[61] Y. S. Kachanov and V. Y. Levchenko, The resonant interaction of disturbances at laminar-turbulent transition in a boundary layer, J. Fluid Mech. 138, 209 (1984).

[62] T. Herbert, Secondary instabilities of boundary layers, Annu. Rev. Fluid Mech. 20, 487 (1988).

[63] U. Rist and H. F. Fasel, Direct numerical simulation of controlled transition in a flat-plate boundary layer, J. Fluid Mech. 298, 211 (1995).

[64] S. Berlin, M. Wiegel, and D. Henningson, Numerical and experimental investigations of oblique boundary layer transition, J. Fluid Mech. 393, 23 (1999).

[65] S. Nagarajan, S. K. Lele, and J. H. Ferziger, Leading-edge effects in bypass transition, J. Fluid Mech. 572, 471 (2007).

[66] V. Ovchinnikov, M. M. Choudhari, and U. Piomelli, Numerical simulations of boundary-layer bypass transition due to high-amplitude free-stream turbulence, J. Fluid Mech. 613, 135 (2008).

[67] R. G. Jacobs and P. A. Durbin, Simulations of bypass transition, J. Fluid Mech. 428, 185 (2001).

[68] D. S. Henningson, A. Lundbladh, and A. V. Johansson, A mechanism for bypass transition from localized disturbances in wall-bounded shear flows, J. Fluid Mech. 250, 169 (1993).

[69] P. Andersson, M. Berggren, and D. S. Henningson, Optimal disturbances and bypass transition in boundary layers, Phys. Fluids 11, 134 (1999).

[70] P. Corbett and A. Bottaro, Optimal perturbations for boundary layers subject to stream-wise pressure gradient, Phys. Fluids 12, 120 (2000).

[71] A. Tumin and E. Reshotko, Optimal disturbances in compressible boundary layers, AIAA J. 41, 2357 (1984).

[72] S. Cherubini, J.-C. Robinet, A. Bottaro, and P. De Palma, Optimal wave packets in a boundary layer and initial phases of a turbulent spot, J. Fluid Mech. 656, 231 (2010).

[73] D. R. Williams, H. Fasel, and F. R. Hama, Experimental determination of the three-dimensional vorticity field in the boundary-layer transition process, J. Fluid Mech. 149, 179 (1984).

[74] S. Bake, H. H. Fernholz, and Y. S. Kachanov, Resemblance of K- and N-regimes of boundary-layer transition at late stages, Eur. J. Mech. B/Fluids 19, 1 (2000).

[75] T. A. Zaki and P. A. Durbin, Mode interaction and the bypass route to transition, J. Fluid Mech. 531, 85 (2005).

[76] A. M. Yaglom, Hydrodynamic Instability and Transition to Turbulence, Fluid Mechanics and Its Applications (Springer, Berlin, 2012), pp. 465-600.

[77] P. A. Elofsson and P. H. Alfredsson, An experimental study of oblique transition in plane Poiseuille flow, J. Fluid Mech. 358, 177 (1998).

[78] A. D. D. Craik, Non-linear resonant instability in boundary layers, J. Fluid Mech. 50, 393 (1971).

[79] P. Ricco and X. Wu, Response of a compressible laminar boundary layer to free-stream vortical disturbances, J. Fluid Mech. 587, 97 (2007).

[80] U. Rist and U. Maucher, Direct numerical simulation of 2-D and 3-D instability waves in a laminar separation bubble, in Application of Direct and Large Eddy Simulation to Transition and Turbulence (AGARD, France, 1994), pp. 34-1-34-7, http://www.dtic.mil/dtic/tr/fulltext/u2/a292121.pdf.

[81] R. D. Joslin, C. L. Streett, and C.-L. Chang, Spatial direct numerical simulation of boundary-layer transition mechanisms: Validation of PSE theory, Theor. Comput. Fluid Dyn. 4, 271 (1993).

[82] B. F. Armaly, F. Durst, J. C. F. Pereira, and B. Schönung, Experimental and theoretical investigation of backward-facing step flow, J. Fluid Mech. 127, 473 (1983).

[83] G. Biswa, M. Breuer, and F. Durst, Backward-facing step flows for various expansion ratios at low and moderate Reynolds numbers, Trans. ASME: J. Fluids Eng. 126, 362 (2004).

[84] R. E. Kelly, On the resonant interaction of neutral disturbances in two inviscid shear flows, J. Fluid Mech. 31, 789 (1968).

[85] M. B. Zelman and I. I. Maslennikova, Tollmien-Schlichting-wave resonant mechanism for subharmonictype transition, J. Fluid Mech. 252, 449 (1993).

[86] T. C. Corke and R. A. Mangano, Resonant growth of three-dimensional modes in transitioning Blasius boundary layers, J. Fluid Mech. 209, 93 (1989). 
[87] V. I. Borodulin, Y. S. Kachanov, and D. B. Koptsev, Experimental study of resonant interactions of instability waves in a self-similar boudary layer with an adverse pressure gradient: I. Tuned resonances, J. Turbulence 3, N62 (2002).

[88] V. I. Borodulin, Y. S. Kachanov, and D. B. Koptsev, Experimental study of resonant interactions of instability waves in a self-similar boudary layer with an adverse pressure gradient: III. Broadband disturbances, J. Turbulence 3, N64 (2002).

[89] T. C. Corke, Three-dimensional mode growth in boundary layers with tuned and detuned subharmonic resonance, Philos. Trans. R. Soc. London, Ser. A 352, 453 (1995).

[90] X. Wu and P. Moin, Direct numerical simulation of turbulence in a nominally zero-pressure-gradient flat-plate boundary layer, J. Fluid Mech. 630, 5 (2009).

[91] X. Wu and P. Moin, Transitional and turbulent boundary layer with heat transfer, Phys. Fluids 22, 085105 (2010).

[92] T. Sayadi, C. W. Hamman, and P. Moin, Direct numerical simulation of H-type and K-type transition to turbulence, Center for Turbulence Research, Annual Research Briefs (CTR, Stanford University, Stanford, California, 2011), pp. 109-121, https://web.stanford.edu/group/ctr/ResBriefs/2011/10_sayadi-color.pdf.

[93] T. Sayadi, C. W. Hamman, and P. Moin, Direct numerical simulation of complete H-type and K-type transitions with implications for the dynamics of turbulent boundary layers, J. Fluid Mech. 724, 480 (2013).

[94] P. Schlatter and R. Örlü, Assessment of direct numerical simulation data of turbulent boundary layers, J. Fluid Mech. 659, 116 (2010).

[95] J. Jimenez, S. Hoyas, M. P. Simens, and Y. Mizuno, Turbulent boundary layers and channels at moderate Reynolds numbers, J. Fluid Mech. 657, 335 (2010).

[96] X. Gloerfelt and F. Margnat, Effect of Mach number on boundary layer noise, in 20th AIAA/CEAS Aeroacoustics Conference, 16-20 June, 2014, Atlanta, Georgia (AIAA, Reston, VA, 2014), paper AIAA 2014-3291.

[97] X. Gloerfelt and E. Cohen, Influence of pressure gradients and Reynolds number on wall-pressure wavenumber-frequency spectra, in 22nd AIAA/CEAS Aeroacoustics Conference, 30 May-1 June, 2016, Lyon, France (AIAA, Reston, VA, 2016), paper AIAA 2016-2910. 